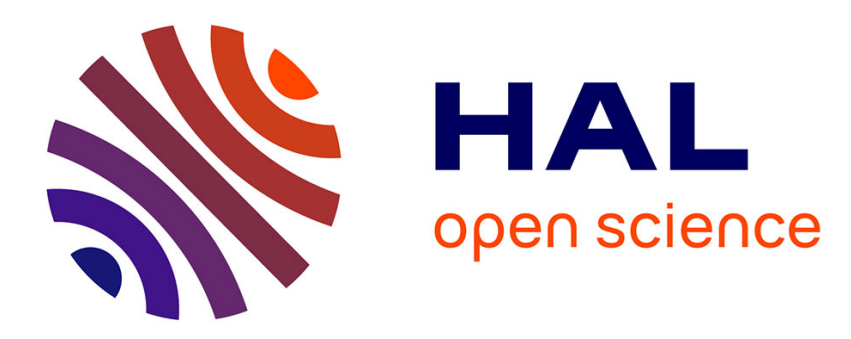

\title{
Theoretical study of a multi-dimensional pressureless model with unilateral constraint
}

Florent Berthelin

\section{To cite this version:}

Florent Berthelin. Theoretical study of a multi-dimensional pressureless model with unilateral constraint. SIAM Journal on Mathematical Analysis, 2017, 49 (3), pp.2287-2320. hal-01313258

\section{HAL Id: hal-01313258 \\ https://hal.science/hal-01313258}

Submitted on 9 May 2016

HAL is a multi-disciplinary open access archive for the deposit and dissemination of scientific research documents, whether they are published or not. The documents may come from teaching and research institutions in France or abroad, or from public or private research centers.
L'archive ouverte pluridisciplinaire HAL, est destinée au dépôt et à la diffusion de documents scientifiques de niveau recherche, publiés ou non, émanant des établissements d'enseignement et de recherche français ou étrangers, des laboratoires publics ou privés. 


\title{
Theoretical study of a multi-dimensional pressureless model with unilateral constraint
}

\author{
F. Berthelin
}

\author{
Laboratoire J. A. Dieudonné, UMR 7351 CNRS, \\ Université de Nice Sophia-Antipolis, Parc Valrose, \\ 06108 Nice cedex 2, France \\ \& Project Team COFFEE, INRIA Sophia Antipolis, France \\ e-mail: Florent.Berthelin@unice.fr
}

\begin{abstract}
We extend to multi-dimension the study of a pressureless model of gas system with unilateral constraint. Several difficulties are added with respect to the one-dimensional case. First, the geometry of the dynamics of blocks cannot be conserved and to solve this problem, a splitting with respect to the various directions is done. This leads to approximations of solutions for special initial data. Besides, the stability of the solutions is also quite different from the one-dimensional case. We finaly get the existence and the stability of solutions.
\end{abstract}

Key-words: conservation laws with constraint - pressureless gas - sticky blocks - splitting dynamics

Mathematics Subject Classification: 35L87, 35L65, 76N10, 35A35, 35D30, $76 \mathrm{~T} 10$ 


\section{Contents}

1 Introduction $\quad 2$

2 Discrete blocks dynamics 4

2 Definition of blocks . . . . . . . . . . . . . . . . 4

2 Continuous dynamics . . . . . . . . . . . . . . . 5

2 Discrete approximations in the free dynamics case . . . . . . . 8

2 Discrete approximations in the constraint case . . . . . . . . . . 12

2 Discrete block approximations and BV estimates . . . . . . . . . 13

3 Discretization and stability $\quad 16$

3 Discretization with blocks . . . . . . . . . . . . . . 16

3 Stability Theorem . . . . . . . . . . . . . . . 20

3 Limit of approximate solutions . . . . . . . . . . . . . . . 25

4 Existence result $\quad 27$

$4 \quad$ Definition of $\rho \pi$ for $\pi$ in the class $\mathcal{M L} \ldots \ldots . . . . .27$

4 The exclusion relation $\rho \pi=\pi$ for solutions with $\pi$ in the class

$\mathcal{M L} \ldots \ldots \ldots \ldots \ldots . \ldots \ldots$

4 Existence of solutions . . . . . . . . . . . . . . . . . . 28

\section{Introduction}

Constraints models have been developed these last years in order to impose some bounds in hyperbolic models. In [8], [2], the following one-dimensional model was studied.

$$
\left\{\begin{array}{l}
\partial_{t} \rho+\partial_{x}(\rho u)=0 \\
\partial_{t}(\rho u)+\partial_{x}\left(\rho u^{2}+\pi\right)=0
\end{array}\right.
$$

with the constraints

$$
0 \leq \rho \leq 1, \quad \pi \geq 0
$$

and the exclusion relation

$$
\rho \pi=\pi .
$$

In [4], an approaching model but with other technical difficulties has been studied. See also [3] for a numerical version of this kind of problems. There are a lot of domains in which constraints models take place. For example, this kind of models have allowed to get better models in trafic flows since the paper [6]. After this paper, some improvements of the model have been done in [7] and [5]. Other hyperbolic problems with constraints have been studied in [1], [13], [15], [16]. Notice also that in [17], the same problem but with viscosity was studied. And in that direction, the limit of barotropic compressible NavierStokes to constraint Navier-Stokes was done in [11] for 1D case and in [18] for the multi-D case. Existence and properties of the system of pressureless gas have been studied in [14], [9], [12].

In this paper, we want to extend the existence and stability result of [2] in multi-dimension. An important tool for this result is the sticky block dynamics. In dimension one, when two blocks collide, they form a new block and the dynamics of blocks is easy. In multi-dimension, a geometric problem appears since when two rectangular parallelepipeds collide, they do not form 
a rectangular parallelepiped. An idea of this paper is to make, on same time interval, a splitting with respect to the various directions of space. Thus on each time interval, we do vary only one direction then, on the next interval, another direction and so on to keep the geometry at each collision. Then by letting the time step going to 0 and thereby forcing the splitting to be more rapid, we hope to find the limit of the speed on any directions. The purpose of this paper is to achieve this approach and prove that it works.

Furthermore, it will give only approximations of solutions instead of solutions for block initial data. Then, the stability and existence of solutions will require additionnal steps than in the one-D case to work.

In order to simplify the presentation, we will detail the 2D-case, but the demarch and proof are the same in any dimension. We will consider the following model with constraint in two dimensions which is the natural extension of (1.1)-(1.3):

$$
\left\{\begin{array}{l}
\partial_{t} \rho+\partial_{x}(\rho u)+\partial_{y}(\rho v)=0 \\
\partial_{t}(\rho u)+\partial_{x}\left(\rho u^{2}+\pi_{1}\right)+\partial_{y}(\rho u v)=0 \\
\partial_{t}(\rho v)+\partial_{x}(\rho u v)+\partial_{y}\left(\rho v^{2}+\pi_{2}\right)=0
\end{array}\right.
$$

with the constraints

$$
0 \leq \rho \leq 1, \quad \pi_{1} \geq 0, \pi_{2} \geq 0,
$$

and the exclusion relations

$$
\rho \pi_{1}=\pi_{1}, \quad \rho \pi_{2}=\pi_{2} .
$$

We will also consider initial data

$$
\rho(0, x, y)(1, u(0, x, y), v(0, x, y))=\rho^{0}(x, y)\left(1, u^{0}(x, y), v^{0}(x, y)\right) .
$$

Let us define precisely the weak solutions we shall consider. We look for solutions satisfying

$$
\begin{gathered}
\rho \in L_{t}^{\infty}(] 0, \infty\left[, L_{x y}^{\infty}\left(\mathbb{R}^{2}\right) \cap L_{x y}^{1}\left(\mathbb{R}^{2}\right)\right) \cap C_{t}\left(\left[0, \infty\left[, L_{w *}^{\infty}\left(\mathbb{R}^{2}\right)\right),\right.\right. \\
u, v \in L_{t}^{\infty}(] 0, \infty\left[, L_{x y}^{\infty}\left(\mathbb{R}^{2}\right)\right), \\
\pi_{1}, \pi_{2} \in \mathcal{M}_{l o c}\left(\left[0, \infty\left[\times \mathbb{R}^{2}\right) .\right.\right.
\end{gathered}
$$

Hence, (1.4), (1.7) must be satisfied in the sense of distributions: for all $\varphi \in C_{c}^{\infty}\left(\left[0,+\infty\left[\times \mathbb{R}^{2}\right)\right.\right.$,

$$
\begin{gathered}
\int_{[0,+\infty[} \iint_{\mathbb{R}^{2}}\left(\rho \partial_{t} \varphi+\rho u \partial_{x} \varphi+\rho v \partial_{y} \varphi\right) d x d y d t \\
+\iint_{\mathbb{R}^{2}} \rho^{0}(x, y) \varphi(0, x, y) d x d y=0 \\
\int_{[0,+\infty[} \iint_{\mathbb{R}^{2}}\left(\rho u \partial_{t} \varphi+\rho u^{2} \partial_{x} \varphi+\rho u v \partial_{y} \varphi\right) d x d y d t \\
+\int_{[0,+\infty[} \iint_{\mathbb{R}^{2}} \partial_{x} \varphi \pi_{1}+\iint_{\mathbb{R}^{2}}\left(\rho^{0} u^{0}\right)(x, y) \varphi(0, x, y) d x d y=0,
\end{gathered}
$$


and

$$
\begin{aligned}
& \int_{[0,+\infty[} \iint_{\mathbb{R}^{2}}\left(\rho v \partial_{t} \varphi+\rho u v \partial_{x} \varphi+\rho v^{2} \partial_{y} \varphi\right) d x d y d t \\
& \quad+\int_{[0,+\infty[} \iint_{\mathbb{R}^{2}} \partial_{y} \varphi \pi_{2}+\iint_{\mathbb{R}^{2}}\left(\rho^{0} v^{0}\right)(x, y) \varphi(0, x, y) d x d y=0 .
\end{aligned}
$$

The constraint (1.6) has also to be understood in a weak sense in the case where the product $\rho \pi$ is not defined. We will have a discussion about this in section 4 and we will define a subspace in which the product is well defined. As we say previously, all the new arguments we introduce to solve the 2D-case can be used the same way in multi-D case. Thus, for example, we can adapt easily the study of this paper for example for the system in dimension three:

$$
\left\{\begin{array}{l}
\partial_{t} \rho+\partial_{x}(\rho u)+\partial_{y}(\rho v)+\partial_{z}(\rho w)=0 \\
\partial_{t}(\rho u)+\partial_{x}\left(\rho u^{2}+\pi_{1}\right)+\partial_{y}(\rho u v)+\partial_{z}(\rho u w)=0 \\
\partial_{t}(\rho v)+\partial_{x}(\rho u v)+\partial_{y}\left(\rho v^{2}+\pi_{2}\right)+\partial_{z}(\rho v w)=0 \\
\partial_{t}(\rho w)+\partial_{x}(\rho u w)+\partial_{y}(\rho v w)+\partial_{z}\left(\rho w^{2}+\pi_{3}\right)=0
\end{array}\right.
$$

with the constraints

$$
0 \leq \rho \leq 1, \quad \pi_{1} \geq 0, \pi_{2} \geq 0, \pi_{3} \geq 0,
$$

and the exclusion relations

$$
\rho \pi_{1}=\pi_{1}, \quad \rho \pi_{2}=\pi_{2}, \quad \rho \pi_{3}=\pi_{3} .
$$

The paper is organized as follows. In section 2 , we will study particular solutions and approximations of solutions in the class of blocks functions. In section 3, we prove a result of discretization of general initial data by blocks and stability results of solutions and approximate solutions. Finally, in section 4 , we study a space in which the constraint can be taken in a classical sense and conclude to the existence result.

\section{Discrete blocks dynamics}

\section{Definition of blocks}

We give here several definitions we are going to use in the following of the paper.

Definition 2.1 We call block initial data a volume fraction $\rho^{0}(x, y)$ with a momentum density $\rho^{0}(x, y)\left(u^{0}(x, y), v^{0}(x, y)\right)$ of the form

$$
\rho^{0}(x, y)\left(1, u^{0}(x, y), v^{0}(x, y)\right)=\sum_{i=-I}^{I^{\prime}} \sum_{j=-J}^{J^{\prime}} \rho_{i j}\left(1, u_{i j}, v_{i j}\right) \mathbb{I}_{(x, y) \in P_{i j}},
$$

where

$$
\mathbb{I}_{(x, y) \in P_{i j}}=\mathbb{I}_{a_{i j} \leq x \leq b_{i j}} \mathbb{I}_{c_{i j} \leq y \leq d_{i j}},
$$

with $I, I^{\prime}, J, J^{\prime} \in \mathbb{N}$ and, for $-I \leq i \leq I^{\prime},-J \leq j \leq J^{\prime}, \rho_{i j} \in\{0,1\}$, $a_{i j}, b_{i j}, c_{i j}, d_{i j}, u_{i j}, v_{i j} \in \mathbb{R}$ such that $\bar{b}_{i j} \leq \bar{a}_{i+1, j}$ and $\overline{d_{i j}} \leq \bar{c}_{i, j+1}$. 
Definition 2.2 We call continuous block a volume fraction $\rho(t, x, y)$ with a momentum density $\rho(t, x, y)(u(t, x, y), v(t, x, y))$ of the form

$$
\rho(t, x, y)(1, u(t, x, y), v(t, x, y))=\sum_{i=-I}^{I^{\prime}} \sum_{j=-J}^{J^{\prime}} \rho_{i j}\left(1, u_{i j}, v_{i j}\right) \mathbb{1}_{(x, y) \in P_{i j}(t)},
$$

where

$$
\mathbb{I}_{(x, y) \in P_{i j}(t)}=\mathbb{I}_{a_{i j}(t) \leq x \leq b_{i j}(t)} \mathbb{I}_{c_{i j}(t) \leq y \leq d_{i j}(t)},
$$

with $I, I^{\prime}, J, J^{\prime} \in \mathbb{N}$ and, for $-I \leq i \leq I^{\prime},-J \leq j \leq J^{\prime}, \rho_{i j} \in\{0,1\}, u_{i j}, v_{i j} \in \mathbb{R}$ and $a_{i j}, b_{i j}, c_{i j}, d_{i j}: \mathbb{R} \rightarrow \mathbb{R}$ such that $b_{i j} \leq a_{i+1, j}$ and $d_{i j} \leq c_{i, j+1}$.

Definition 2.3 Let us given $\Delta t, \Delta x, \Delta y>0$. We call discrete block a volume fraction $\rho(t, x, y)$ with a momentum density $\rho(t, x, y)(u(t, x, y), v(t, x, y))$ of the form

$$
\rho(t, x, y)(1, u(t, x, y), v(t, x, y))=\sum_{i=-I}^{I^{\prime}} \sum_{j=-J}^{J^{\prime}} \sum_{l=0}^{+\infty} \rho_{i j l}\left(1, u_{i j l}, v_{i j l}\right) \mathbb{I}_{(t, x, y) \in P_{i j l}(t)},
$$

where

$$
\mathbb{I}_{(t, x, y) \in P_{i j l}}=\mathbb{I}_{l \Delta t \leq t<(l+1) \Delta t} \mathbb{I}_{a_{i j l}+i \Delta x \leq x<a_{i j l}+(i+1) \Delta x} \mathbb{I}_{b_{i j l}+j \Delta y \leq y<b_{i j l}+(j+1) \Delta y},
$$

with $I, I^{\prime}, J, J^{\prime} \in \mathbb{N}$ and, for $-I \leq i \leq I^{\prime},-J \leq j \leq J^{\prime}, l \in \mathbb{N}, \rho_{i j l} \in\{0,1\}$, $a_{i j l}, b_{i j l}, c_{i j l}, d_{i j l}, u_{i j l}, v_{i j l} \in \mathbb{R}$ such that $a_{i j l}+\Delta x \leq a_{i+1, j l}$ and $b_{i j l}+\Delta y \leq b_{i, j+1, l}$.

Remark 2.1 To simplify the presentation, we can assume that $I=J=0$ which is just a translation of indices and $I^{\prime}=J^{\prime}$ by adding zero terms to have the same number of terms. In the following, we may sometimes use this change of notations by setting $N-1=I^{\prime}=J^{\prime}$.

\section{Continuous dynamics}

We start first by studying the free dynamics, we mean when constraints doesn't act. It leads to the study of pressureless dynamics equations in dimension two, which are given by

$$
\left\{\begin{array}{l}
\partial_{t} \rho+\partial_{x}(\rho u)+\partial_{y}(\rho v)=0 \\
\partial_{t}(\rho u)+\partial_{x}\left(\rho u^{2}\right)+\partial_{y}(\rho u v)=0 \\
\partial_{t}(\rho v)+\partial_{x}(\rho u v)+\partial_{y}\left(\rho v^{2}\right)=0
\end{array}\right.
$$

We prove now that continuous blocks with functions $a(t), b(t)$ adapted with a constant velocity $(u, v)$ are solutions to the free dynamics.

Proposition 2.4 Let $u, v, a_{0}, b_{0} \in \mathbb{R}$ and $c, d>0$. The functions

$$
\tilde{\rho}(1, \tilde{u}, \tilde{v})(t, x, y)=(1, u, v) \mathbb{I}_{0 \leq t} \mathbb{I}_{a(t) \leq x \leq a(t)+c} \mathbb{I}_{b(t) \leq y \leq b(t)+d},
$$

where $a(t)=a_{0}+u t$ and $b(t)=b_{0}+v t$, are solution of (2.23) in the distributional sense with the initial data

$$
(1, u, v) \mathbb{I}_{a_{0} \leq x \leq a_{0}+c} \mathbb{I}_{b_{0} \leq y \leq b_{0}+d}
$$


Proof. Let $S: \mathbb{R}^{2} \rightarrow \mathbb{R}$ be a continuous function. Let $\varphi \in C_{c}^{\infty}\left(\left[0,+\infty\left[, \mathbb{R}^{2}\right)\right.\right.$, we want to compute

$$
\int_{0}^{+\infty} \int_{a(t)}^{a(t)+c} \int_{b(t)}^{b(t)+d} S(\tilde{u}, \tilde{v})\left(\partial_{t} \varphi+u \partial_{x} \varphi+v \partial_{y} \varphi\right)(t, x, y) d y d x d t
$$

First

$$
\begin{aligned}
& \frac{d}{d t}\left(\int_{a(t)}^{a(t)+c} \int_{b(t)}^{b(t)+d} \varphi(t, x, y) d y d x\right) \\
= & \int_{a(t)}^{a(t)+c} \int_{b(t)}^{b(t)+d} \partial_{t} \varphi(t, x, y) d y d x \\
& +a^{\prime}(t)\left(\int_{b(t)}^{b(t)+d} \varphi(t, a(t)+c, y) d y-\int_{b(t)}^{b(t)+d} \varphi(t, a(t)+c, y) d y\right) \\
& +b^{\prime}(t)\left(\int_{a(t)}^{a(t)+c} \varphi(t, x, b(t)+d) d x-\int_{a(t)}^{a(t)+c} \varphi(t, x, b(t)+d) d x\right),
\end{aligned}
$$

furthermore

$$
\begin{aligned}
& \int_{0}^{+\infty} \int_{a(t)}^{a(t)+c} \int_{b(t)}^{b(t)+d} \partial_{x} \varphi(t, x, y) d y d x d t \\
= & \int_{0}^{+\infty} \int_{b(t)}^{b(t)+d} \varphi(t, a(t)+c, y)-\varphi(t, a(t)+c, y) d y d t
\end{aligned}
$$

and

$$
\begin{aligned}
& \int_{0}^{+\infty} \int_{a(t)}^{a(t)+c} \int_{b(t)}^{b(t)+d} \partial_{y} \varphi(t, x, y) d y d x d t \\
= & \int_{0}^{+\infty} \int_{a(t)}^{a(t)+c} \varphi(t, x, b(t)+d)-\varphi(t, x, b(t)+d) d x d t .
\end{aligned}
$$

Integrating with respect to $t$ the relation (2.25) and using that $a^{\prime}(t)=u$ and $b^{\prime}(t)=v$, we get that

$$
\begin{aligned}
& \int_{0}^{+\infty} \int_{a(t)}^{a(t)+c} \int_{b(t)}^{b(t)+d} S(\tilde{u}, \tilde{v})\left(\partial_{t} \varphi+u \partial_{x} \varphi+v \partial_{y} \varphi\right)(t, x, y) d y d x d t \\
= & -\int_{a_{0}}^{a_{0}+c} \int_{b_{0}}^{b_{0}+d} \varphi(0, x, y) d y d x .
\end{aligned}
$$

Applying this to $S(u, v)=1, S(u, v)=u$ and $S(u, v)=v$, we get the result.

The previous dynamics concerns the evolution of blocks as long as there is no collision between them. Now we consider the case with a collision in the $x$ direction. 
Proposition 2.5 Let $t^{*}, \mu>0, x^{*}, u_{1}, u_{2}, c, d, v \in \mathbb{R}$. The functions

$$
\begin{aligned}
\hat{\rho}(1, \hat{u}, \hat{v})(t, x, y)= & \mathbb{I}_{0 \leq t<t^{*}}\left(\left(1, u_{1}, v\right) \mathbb{I}_{a_{1}(t)-c \leq x \leq a_{1}(t)} \mathbb{I}_{b(t) \leq y \leq b(t)+\mu}\right. \\
& \left.\quad+\left(1, u_{2}, v\right) \mathbb{I}_{a_{2}(t) \leq x \leq a_{2}(t)+d} \mathbb{I}_{b(t) \leq y \leq b(t)+\mu}\right) \\
& +\left(1, u_{f}, v\right) \mathbb{I}_{t^{*} \leq t} \mathbb{I}_{a_{f}(t)-c \leq x \leq a_{f}(t)+d} \mathbb{I}_{b(t) \leq y \leq b(t)+\mu},
\end{aligned}
$$

and the measures

$$
\pi_{1}(t, x, y)= \begin{cases}\delta\left(t-t^{*}\right)\left(u_{1}-u_{f}\right)(x-(z-c)) & \text { if } z-c \leq x \leq z \\ \delta\left(t-t^{*}\right)\left(u_{f}-u_{2}\right)((z+d)-x) & \text { if } z \leq x \leq z+d \\ 0 & \text { otherwise }\end{cases}
$$

and $\pi_{2}=0$, where $a_{1}(t)=x^{*}+u_{1}\left(t-t^{*}\right), a_{2}(t)=x^{*}+u_{2}\left(t-t^{*}\right)$ and $a_{f}(t)=x^{*}+$ $u_{f}\left(t-t^{*}\right)$ (the point $x^{*}$ being the point of collision) with $c u_{1}+d u_{2}=(c+d) u_{f}$, are solution of (1.4), (1.5) and (1.6) in the distributional sense.

Proof. Let $\varphi$ be a test function and $S: \mathbb{R}^{2} \rightarrow \mathbb{R}$ be a continuous function. We have

$$
\begin{aligned}
& <\partial_{t}(\hat{\rho} S(\hat{u}, \hat{v}))+\partial_{x}(\hat{\rho} S(\hat{u}, \hat{v}) \hat{u})+\partial_{y}(\hat{\rho} S(\hat{u}, \hat{v}) \hat{v}), \varphi> \\
= & -\int_{0}^{t^{*}} \int_{a_{1}(t)-c}^{a_{1}(t)} \int_{b(t)}^{b(t)+\mu} S\left(u_{1}, v\right)\left(\partial_{t} \varphi+u_{1} \partial_{x} \varphi+v \partial_{y} \varphi\right) d y d x d t \\
& -\int_{0}^{t^{*}} \int_{a_{2}(t)}^{a_{2}(t)+d} \int_{b(t)}^{b(t)+\mu} S\left(u_{2}, v\right)\left(\partial_{t} \varphi+u_{2} \partial_{x} \varphi+v \partial_{y} \varphi\right) d y d x d t \\
& -\int_{t^{*}}^{+\infty} \int_{a_{f}(t)-c}^{a_{f}(t)+d} \int_{b(t)}^{b(t)+\mu} S\left(u_{f}, v\right)\left(\partial_{t} \varphi+u_{f} \partial_{x} \varphi+v \partial_{y} \varphi\right) d y d x d t
\end{aligned}
$$

Notice that

$$
\begin{aligned}
& \frac{d}{d t}\left(\int_{a_{1}(t)-c}^{a_{1}(t)} \int_{b(t)}^{b(t)+\mu} \varphi(t, x, y) d y d x\right) \\
= & \int_{a_{1}(t)-c}^{a_{1}(t)} \int_{b(t)}^{b(t)+\mu} \partial_{t} \varphi(t, x, y) d y d x \\
& +\int_{b(t)}^{b(t)+\mu}\left(\varphi\left(t, a_{1}(t), y\right)-\varphi\left(t, a_{1}(t)-c, y\right)\right) u_{1} d y \\
& +\int_{a_{1}(t)-c}^{a_{1}(t)}(\varphi(t, x, b(t)+\mu)-\varphi(t, x, b(t))) v d x,
\end{aligned}
$$

then we get

$$
\begin{aligned}
& \int_{0}^{t^{*}} \int_{a_{1}(t)-c}^{a_{1}(t)} \int_{b(t)}^{b(t)+\mu} S\left(u_{1}, v\right) \partial_{t} \varphi(t, x, y) d y d x d t \\
= & \int_{a_{1}\left(t^{*}\right)-c}^{a_{1}\left(t^{*}\right)} \int_{b\left(t^{*}\right)}^{b\left(t^{*}\right)+\mu} S\left(u_{1}, v\right) \varphi\left(t^{*}, x, y\right) d y d x
\end{aligned}
$$




$$
\begin{aligned}
& -\int_{0}^{t^{*}} \int_{b(t)}^{b(t)+\mu} S\left(u_{1}, v\right) u_{1}\left(\varphi\left(t, a_{1}(t), y\right)-\varphi\left(t, a_{1}(t)-c, y\right)\right) d y d t \\
& -\int_{0}^{t^{*}} \int_{a_{1}(t)-c}^{a_{1}(t)} S\left(u_{1}, v\right) v(\varphi(t, x, b(t)+\mu)-\varphi(t, x, b(t))) d x d t \\
= & \int_{a_{1}\left(t^{*}\right)-c}^{a_{1}\left(t^{*}\right)} \int_{b\left(t^{*}\right)}^{b\left(t^{*}\right)+\mu} S\left(u_{1}, v\right) \varphi\left(t^{*}, x, y\right) d y d x \\
& -\int_{0}^{t^{*}} \int_{a_{1}(t)-c}^{a_{1}(t)} \int_{b(t)}^{b(t)+\mu} S\left(u_{1}, v\right) u_{1} \partial_{x} \varphi(t, x, y) d y d x d t \\
& -\int_{0}^{t^{*}} \int_{a_{1}(t)-c}^{a_{1}(t)} \int_{b(t)}^{b(t)+\mu} S\left(u_{1}, v\right) v \partial_{y} \varphi(t, x, y) d y d x d t .
\end{aligned}
$$

We have similar equations for both terms (2.28) and (2.29) and we get

$$
\begin{aligned}
<\partial_{t}(\hat{\rho} S(\hat{u}, \hat{v}))+\partial_{x}(\hat{\rho} S(\hat{u}, \hat{v}) \hat{u})+\partial_{y}(\hat{\rho} S(\hat{u}, \hat{v}) \hat{v}), \varphi> \\
=-\int_{b\left(t^{*}\right)}^{b\left(t^{*}\right)+\mu}\left(\int_{x^{*}-c}^{x^{*}}\left(S\left(u_{1}\right)-S\left(u_{f}\right)\right) \varphi\left(t^{*}, x, y\right) d x\right. \\
\left.\quad+\int_{x^{*}}^{x^{*}+d}\left(S\left(u_{2}\right)-S\left(u_{f}\right)\right) \varphi\left(t^{*}, x, y\right) d x\right) d y
\end{aligned}
$$

For $S(u)=1$, it gives $\partial_{t} \hat{\rho}+\partial_{x}(\hat{\rho} \hat{u})+\partial_{y}(\hat{\rho} \hat{v})=0$, for $S(u, v)=v$, it gives $\partial_{t}(\hat{\rho} \hat{v})+\partial_{x}(\hat{\rho} \hat{u} \hat{v})+\partial_{y}\left(\hat{\rho} \hat{v}^{2}\right)=0$ and for $S(u)=u$, we get $\partial_{t}(\hat{\rho} \hat{u})+\partial_{x}\left(\hat{\rho} \hat{u}^{2}+\right.$ $\left.\pi_{1}\right)+\partial_{y}(\hat{\rho} \hat{u} \hat{v})=0$ where $\pi_{1}$ is defined by (2.26). Notice that $\pi_{1} \geq 0$ and that the constraints relations are satisfied.

Remark 2.2 If we do the same with a shock in the $y$ direction, it gives a term $\pi_{2} \neq 0$.

\section{Discrete approximations in the free dynamics case}

Let $\Delta t, \Delta x$ and $\Delta y$ be non-negative reals. We prove here that we can approximate the solution of Proposition 2.4 with discrete blocks.

Proposition 2.6 Let $u, v, a_{0}, b_{0} \in \mathbb{R}$ and $c, d>0$. Then there exists discrete blocks $\left(\rho_{N}, \rho_{N} u_{N}, \rho_{N} v_{N}\right)$ with initial data $\mathbb{I}_{a_{0} \leq x \leq a_{0}+c} \mathbb{I}_{b_{0} \leq y \leq b_{0}+d}(1, u, v)$ such that

$$
\left\{\begin{array}{l}
\partial_{t} \rho_{N}+\partial_{x}\left(\rho_{N} u_{N}\right)+\partial_{y}\left(\rho_{N} v_{N}\right) \rightarrow 0 \\
\partial_{t}\left(\rho_{N} u_{N}\right)+\partial_{x}\left(\rho_{N} u_{N}^{2}\right)+\partial_{y}\left(\rho_{N} u_{N} v_{N}\right) \rightarrow 0 \\
\partial_{t}\left(\rho_{N} v_{N}\right)+\partial_{x}\left(\rho_{N} u_{N} v_{N}\right)+\partial_{y}\left(\rho_{N} v_{N}^{2}\right) \rightarrow 0
\end{array}\right.
$$

in the distributional sense.

Definition 2.7 We first define the dynamics of blocks we are going to use in this case. Let $u, v, a_{0}, b_{0} \in \mathbb{R}$ and $c, d>0$. Let $N \in \mathbb{N}^{*}$. We take $\Delta x=c / N$, $\Delta y=d / N$ and $\Delta t=1 / N$. The key idea is to perform a splitting in time. During a time $\Delta t$, we make only act the $x$ direction movement, then during the following $\Delta t$ time, we make only act the $y$ direction movement and so on with alternatively a movement on $x$ direction and on $y$ direction. 
More precisely, starting from $a_{0}$ and $b_{0}$, we construct the sequences $\left(a_{n}\right)_{n}$ and $\left(b_{n}\right)_{n}$ as

$$
a_{2 k+1}=a_{0}+\left[\frac{2(k+1) u \Delta t}{\Delta x}\right] \Delta x, \quad b_{2 k+1}=b_{2 k},
$$

and

$$
b_{2 k+2}=b_{0}+\left[\frac{2(k+1) v \Delta t}{\Delta y}\right] \Delta y, \quad a_{2 k+2}=a_{2 k+1} .
$$

At time $t=(2 k+1) \Delta t$, we make a jump for the block in the $x$ direction, and at time $t=(2 k+2) \Delta t$, we make a jump for the block in the $y$ direction, staying on the fixed grid at level $N$ and taking an approximation of the movement. Then we consider the approximation given by the following sum of blocks:

$$
\rho_{N}\left(1, u_{N}, v_{N}\right)(t, x, y)=\sum_{i, j=0}^{N-1} \sum_{l=0}^{+\infty}(1, u, v) \mathbb{1}_{(t, x, y) \in P_{i j l}}
$$

where

$$
\mathbb{I}_{(t, x, y) \in P_{i j l}}=\mathbb{I}_{l \Delta t \leq t<(l+1) \Delta t} \mathbb{I}_{a_{l}+i \Delta x \leq x<a_{l}+(i+1) \Delta x} \mathbb{I}_{b_{l}+j \Delta y \leq y<b_{l}+(j+1) \Delta y}
$$

We first start by proving the two following technical Lemmas.

Lemma 2.8 We use the discrete blocks constructed in Definition 2.7 and the associated notations. We set

$$
a_{\Delta}(t)=\sum_{l=0}^{+\infty} a_{l} \mathbb{I}_{l \Delta t \leq t<(l+1) \Delta t} .
$$

Then we have

$$
\left|a(t)-a_{\Delta}(t)\right| \leq|u| \Delta t+\Delta x
$$

and

$$
\left|b(t)-b_{\Delta}(t)\right| \leq|v| \Delta t+\Delta y
$$

Proof. Using that $2(k+1) u \Delta t-\Delta x<\left[\frac{2(k+1) u \Delta t}{\Delta x}\right] \Delta x \leq 2(k+1) u \Delta t$, for $t \in[(2 k+1) \Delta t,(2 k+3) \Delta t[$, we have

$$
\begin{aligned}
\left|a(t)-a_{\Delta}(t)\right| & =\left|a_{2 k+1}-a_{0}-u t\right|=\left|\left[\frac{2(k+1) u \Delta t}{\Delta x}\right] \Delta x-u t\right| \\
& \leq|u||2(k+1) \Delta t-t|+\Delta x \\
& \leq|u| \Delta t+\Delta x .
\end{aligned}
$$

Then, for any $t \geq 0$, we get

$$
\left|a(t)-a_{\Delta}(t)\right| \leq|u| \Delta t+\Delta x .
$$

Similarly, we have $\left|b(t)-b_{\Delta}(t)\right| \leq|v| \Delta t+\Delta y$. 
Lemma 2.9 We use the discrete blocks constructed and the associated notations of Definition 2.7 and Lemma 2.8. Setting, for any test function $\varphi \in$ $C_{c}^{\infty}\left(\left[0,+\infty\left[, \mathbb{R}^{2}\right)\right.\right.$

$$
A(\varphi)=\sum_{l=0}^{+\infty} \int_{l \Delta t}^{(l+1) \Delta t} \int_{a(t)}^{a(t)+c} \int_{b(t)}^{b(t)+d} \varphi(t, x, y) d y d x d t
$$

and

$$
A_{N}(\varphi)=\sum_{i, j=0}^{N-1} \sum_{l=0}^{+\infty} \int_{l \Delta t}^{(l+1) \Delta t} \int_{a_{l}+i \Delta x}^{a_{l}+(i+1) \Delta x} \int_{b_{l}+j \Delta y}^{b_{l}+(j+1) \Delta y} \varphi(t, x, y) d y d x d t
$$

Then we have $A_{N}(\varphi) \rightarrow A(\varphi)$ when $N \rightarrow+\infty$.

Proof. Since $c=N \Delta x$ and $d=N \Delta y$, notice that

$$
A(\varphi)=\sum_{i, j=0}^{N-1} \sum_{l=0}^{+\infty} \int_{l \Delta t}^{(l+1) \Delta t} \int_{a(t)+i \Delta x}^{a(t)+(i+1) \Delta x} \int_{b(t)+j \Delta y}^{b(t)+(j+1) \Delta y} \varphi(t, x, y) d y d x d t .
$$

Let us denote by $T$ a real such that the support in time of $\varphi$ is in $[0, T]$. Denote by $L_{N}$ an integer such that $L_{N} \Delta t \geq T$. We have

$$
\begin{aligned}
A_{N}(\varphi)-A(\varphi)= & \sum_{i, j=0}^{N-1} \sum_{l=0}^{+\infty} \int_{l \Delta t}^{(l+1) \Delta t}\left(\int_{a_{l}+i \Delta x}^{a_{l}+(i+1) \Delta x} \int_{b_{l}+j \Delta y}^{b_{l}+(j+1) \Delta y} \varphi(t, x, y) d y d x\right. \\
& \left.-\int_{a(t)+i \Delta x}^{a(t)+(i+1) \Delta x} \int_{b(t)+j \Delta y}^{b(t)+(j+1) \Delta y} \varphi(t, x, y) d y d x\right) d t \\
= & \sum_{i, j=0}^{N-1} \sum_{l=0}^{L_{N}} \int_{l \Delta t}^{(l+1) \Delta t} \int_{a_{l}+i \Delta x}^{a_{l}+(i+1) \Delta x} \int_{b_{l}+j \Delta y}^{b_{l}+(j+1) \Delta y} \\
& \left(\varphi(t, x, y)-\varphi\left(t, x+a(t)-a_{l}, y+b(t)-b_{l}\right)\right) d y d x d t .
\end{aligned}
$$

Let $\varepsilon>0$. Since $\varphi$ is continuous and has a compact support, there exists $\eta>0$ such that for any $\left(t, x_{1}, y_{1}\right)$ and $\left(t, x_{2}, y_{2}\right)$ in the support of $\varphi$, if $\left|x_{1}-x_{2}\right| \leq \eta$ and $\left|y_{1}-y_{2}\right| \leq \eta$, then $\left|\varphi\left(t, x_{1}, y_{1}\right)-\varphi\left(t, x_{2}, y_{2}\right)\right| \leq \varepsilon$. Let $N_{0} \in \mathbb{N}^{*}$ be such that $N_{0}$ is greater than $(|u|+c) / \eta$ and $(|v|+d) / \eta$. Let $N \in N^{*}$ be greater than $N_{0}$. Now

$$
\left|a(t)-a_{\Delta}(t)\right| \leq|u| \Delta t+\Delta x=|u| \frac{1}{N}+\frac{c}{N} \leq \eta
$$

and $\left|b(t)-b_{\Delta}(t)\right| \leq|v| \frac{1}{N}+\frac{d}{N} \leq \eta$, therefore

$$
\begin{aligned}
\left|A_{N}(\varphi)-A(\varphi)\right| & \leq \sum_{i, j=0}^{N-1} \sum_{l=0}^{L_{N}} \int_{l \Delta t}^{(l+1) \Delta t} \int_{a_{l}+i \Delta x}^{a_{l}+(i+1) \Delta x} \int_{b_{l}+j \Delta y}^{b_{l}+(j+1) \Delta y} \varepsilon d y d x d t \\
& \leq \sum_{i, j=0}^{N-1} \sum_{l=0}^{L_{N}} \int_{l \Delta t}^{(l+1) \Delta t} \Delta x \Delta y \varepsilon d t \\
& \leq N \Delta x N \Delta y L_{N} \Delta \varepsilon \\
& \leq c d T \varepsilon
\end{aligned}
$$


It gives that $A_{N}(\varphi) \rightarrow A(\varphi)$ when $N \rightarrow+\infty$.

We can now conclude to the proof of Proposition 2.6.

Proof of Proposition 2.6. Let $\varphi \in C_{c}^{\infty}\left(\left[0,+\infty\left[, \mathbb{R}^{2}\right)\right.\right.$. The solution $(\tilde{\rho}, \tilde{\rho} \tilde{u}, \tilde{\rho} \tilde{u})$ satisfies

$$
\begin{aligned}
0 & =\int_{0}^{+\infty} \iint_{\mathbb{R}^{2}}\left(\tilde{\rho} \partial_{t} \varphi+\tilde{\rho} \tilde{u} \partial_{x} \varphi+\tilde{\rho} \tilde{v} \partial_{y} \varphi\right) d y d x d t \\
& =\sum_{l=0}^{+\infty} \int_{l \Delta t}^{(l+1) \Delta t} \int_{a(t)}^{a(t)+\Delta x} \int_{b(t)}^{b(t)+\Delta y}\left(\partial_{t} \varphi+u \partial_{x} \varphi+v \partial_{y} \varphi\right) d y d x d t \\
& =A\left(\partial_{t} \varphi\right)+u A\left(\partial_{x} \varphi\right)+v A\left(\partial_{y} \varphi\right) .
\end{aligned}
$$

We also have

$$
\begin{aligned}
& \int_{0}^{+\infty} \iint_{\mathbb{R}^{2}}\left(\rho_{N} \partial_{t} \varphi+\rho_{N} u_{N} \partial_{x} \varphi+\rho_{N} v_{N} \partial_{y} \varphi\right) d y d x d t \\
= & \sum_{i, j=0}^{N-1} \sum_{l=0}^{+\infty} \int_{l \Delta t}^{(l+1) \Delta t} \int_{a_{l}+i \Delta x}^{a_{l}+(i+1) \Delta x} \int_{b_{l}+j \Delta y}^{b_{l}+(j+1) \Delta y}\left(\partial_{t} \varphi+u \partial_{x} \varphi+v \partial_{y} \varphi\right) d y d x d t \\
= & A_{N}\left(\partial_{t} \varphi\right)+u A_{N}\left(\partial_{x} \varphi\right)+v A_{N}\left(\partial_{y} \varphi\right) .
\end{aligned}
$$

Since $A\left(\partial_{t} \varphi\right)+u A\left(\partial_{x} \varphi\right)+v A\left(\partial_{y} \varphi\right)=0$, then we get that

$$
A_{N}\left(\partial_{t} \varphi\right)+u A_{N}\left(\partial_{x} \varphi\right)+v A_{N}\left(\partial_{y} \varphi\right) \underset{N \rightarrow+\infty}{\rightarrow} 0
$$

applying the Lemma 2.9 to $\partial_{t} \varphi, \partial_{x} \varphi$ and $\partial_{y} \varphi$. That is to say

$$
\int_{0}^{+\infty} \iint_{\mathbb{R}^{2}}\left(\rho_{N} \partial_{t} \varphi+\rho_{N} u_{N} \partial_{x} \varphi+\rho_{N} v_{N} \partial_{y} \varphi\right) d y d x d t \underset{N t \rightarrow+\infty}{\rightarrow} 0
$$

for any test function $\varphi$.

Since the speeds $u$ and $v$ are constants, they can be put in factor on every terms and then we get also that

$$
\int_{0}^{+\infty} \iint_{\mathbb{R}^{2}}\left(\rho_{N} u_{N} \partial_{t} \varphi+\rho_{N} u_{N}^{2} \partial_{x} \varphi+\rho_{N} v_{N} u_{N} \partial_{y} \varphi\right) d y d x d t \underset{N \rightarrow+\infty}{\rightarrow} 0
$$

and

$$
\int_{0}^{+\infty} \iint_{\mathbb{R}^{2}}\left(\rho_{N} v_{N} \partial_{t} \varphi+\rho_{N} u_{N} v_{N} \partial_{x} \varphi+\rho_{N} v_{N}^{2} \partial_{y} \varphi\right) d y d x d t \underset{N \rightarrow+\infty}{\rightarrow} 0
$$

for any test function $\varphi$. 


\section{Discrete approximations in the constraint case}

We define now the dynamics to approximate the solution of Proposition 2.5 by discrete blocks.

Definition 2.10 First consider that the shock happens during the $x$ direction movement in the splitting.

We still take, for $N \in \mathbb{N}^{*}, \Delta x=c / N, \Delta y=d / N$ and $\Delta t=1 / N$. We start at $t=0$ from a situation where two distincts blocks, the first one with a length $c=P \Delta x$ and a velocity $u_{1}$ located between $\alpha^{1}-P \Delta x$ and $\alpha^{1}$ and the second one with a length $d=Q \Delta x$ and a velocity $u_{2}$ located between $\alpha^{2}$ and $\alpha^{2}+Q \Delta x$. If $0<\frac{\alpha^{2}-\alpha^{1}}{u_{1}-u_{2}} \leq \Delta t$, then a collision has to happen in time $t^{*}=\frac{\alpha^{2}-\alpha^{1}}{u_{1}-u_{2}}$. In order to have the conservation of the mass and a good approximation of the conservation of the momentum, at time $\Delta t$, we replace this by the following situation: a block with a length $(P+Q) \Delta x$ and a velocity $u_{f}=\frac{P u_{1}+Q u_{2}}{P+Q}$ located between $\alpha^{f}-P \Delta x$ and $\alpha^{f}+Q \Delta x$ where

$$
\alpha^{f}=\left[\frac{u_{1} t^{*}+\alpha^{1}+u_{f}\left(\Delta t-t^{*}\right)}{\Delta x}\right] \Delta x .
$$

We have similar formulas for a shock in the $y$ direction remplacing $\Delta x$ by $\Delta y$ and $u$ by $v$.

If the shock is not between 0 and $\Delta t$, but let say between $L \Delta t$ and $(L+1) \Delta t$, we just have to make a translation of these formulas.

We prove now to that the discrete blocks defined previously are approximations of the solution of Proposition 2.5.

Proposition 2.11 We denote by $\left(\hat{\rho}_{N}, \hat{\rho}_{N} \hat{u}_{N}, \hat{\rho}_{N} \hat{v}_{N}\right)$ the discrete blocks constructed in Definition 2.10 (see formula (2.38) for the part of this function which is located at the collision). Then the functions $\left(\hat{\rho}_{N}, \hat{\rho}_{N} \hat{u}_{N}, \hat{\rho}_{N} \hat{v}_{N}\right)$ have the continuous block functions $(\hat{\rho}, \hat{\rho} \hat{u}, \hat{\rho} \hat{v})$ for limit in the distributional sense when $N \rightarrow+\infty$.

Proof. We consider the case of a shock in the $x$ direction with the previous notations. Denote by $L$ (which changes with $\Delta t$, that is to say with $N$ ) the integer such that $t^{*} \in[L \Delta t,(L+1) \Delta t[$, and we notice that the part of the functions located near the collision can be written as

$$
\hat{\rho}_{N}\left(1, \hat{u}_{N}, \hat{v}_{N}\right)(t, x, y)=\left(1, u_{f}, v\right) \mathbb{I}_{L \Delta t \leq t<t^{*}} \mathbb{I}_{(x, y) \in \mathcal{P}}
$$

where

$$
\mathbb{I}_{(x, y) \in \mathcal{P}}=\mathbb{I}_{\alpha^{f}-P \Delta x \leq x<\alpha^{f}+Q \Delta x} \mathbb{I}_{b_{l} \leq y<b_{l}+\mu} .
$$

Notice that before $L \Delta t$ and after $(L+1) \Delta t$, the movement is free and we have studied it already. Notice also that after the shock, the positions $a_{l}^{f}$ of the blocks move as in the free case starting with the new defined positions. 
We consider a test function $\varphi \in C_{c}^{\infty}\left(\left[0,+\infty\left[, \mathbb{R}^{2}\right)\right.\right.$. We have

$$
\begin{aligned}
& \int_{0}^{+\infty} \iint_{\mathbb{R}^{2}}\left(\hat{\rho}_{N} \partial_{t} \varphi+\hat{\rho}_{N} \hat{u}_{N} \partial_{x} \varphi+\hat{\rho}_{N} \hat{v}_{N} \partial_{y} \varphi\right) d y d x d t \\
= & \int_{L \Delta t}^{(L+1) \Delta t} \int_{\alpha^{f}-P \Delta x}^{\alpha^{f}+Q \Delta x} \int_{b_{l}}^{b_{l}+\mu}\left(\partial_{t} \varphi+u \partial_{x} \varphi+v \partial_{y} \varphi\right) d y d x d t \\
& +R_{N}(\varphi),
\end{aligned}
$$

where $R_{N}(\varphi) \rightarrow 0$ corresponding to the part of $\hat{\rho}_{N}$ which follows a free movement and has yet been studied. We will consider the difference with the corresponding terms for $(\hat{\rho}, \hat{\rho} \hat{u}, \hat{v})$. We have then to consider the difference

$$
\begin{aligned}
B_{N}(\varphi)= & \int_{L \Delta t}^{t^{*}} \int_{\alpha^{f}-c}^{\alpha^{f}} \int_{b_{l}}^{b_{l}+\mu}\left(\varphi(t, x, y)-\varphi\left(t, x-\alpha^{f}+a_{1}(t), y\right)\right) d y d x d t \\
& +\int_{L \Delta t}^{t^{*}} \int_{\alpha^{f}}^{\alpha^{f}+d} \int_{b_{l}}^{b_{l}+\mu}\left(\varphi(t, x, y)-\varphi\left(t, x-\alpha^{f}+a_{2}(t), y\right)\right) d y d x d t \\
& +\int_{t^{*}}^{(L+1) \Delta t} \int_{\alpha^{f}-c}^{\alpha^{f}+d} \int_{b_{l}}^{b_{l}+\mu}\left(\varphi(t, x, y)-\varphi\left(t, x-\alpha^{f}+a^{f}(t), y\right)\right) d y d x d t .
\end{aligned}
$$

We have $a_{1}(t)=\alpha^{1}+u_{1}(t-L \Delta), a_{2}(t)=\alpha^{2}+u_{2}(t-L \Delta t)$ and $x^{*}=\alpha^{1}+$ $u_{1}\left(t^{*}-L \Delta t\right)$, then for $t \in[L \Delta t,(L+1) \Delta t[$,

$$
\begin{gathered}
\left|\alpha^{f}-a^{f}(t)\right| \leq\left|u_{f}(t-L \Delta t)\right|+\Delta x \leq\left|u_{f}\right| \Delta t+\Delta x \\
\left|\alpha^{f}-a_{1}(t)\right| \leq\left|\left(u_{1}-u_{f}\right)\left(t^{*}-L \Delta t\right)\right|+\left|u_{f}(\Delta t-t)\right|+\Delta x \leq\left(\left|u_{1}-u_{f}\right|+\left|u_{f}\right|\right) \Delta t+\Delta x
\end{gathered}
$$

and

$\left|\alpha^{f}-a_{2}(t)\right| \leq\left|\left(u_{2}-u_{f}\right)\left(t^{*}-L \Delta t\right)\right|+\left|u_{f}(\Delta t-t)\right|+\Delta x \leq\left(\left|u_{2}-u_{f}\right|+\left|u_{f}\right|\right) \Delta t+\Delta x$.

Then we do as in the free case (for the terms $A_{N}(\varphi)$ ) to get that $B_{N}(\varphi) \rightarrow 0$ when $N \rightarrow+\infty$.

\section{Discrete block approximations and BV estimates}

Starting from an initial block data,

$$
\rho^{0}(x, y)\left(1, u^{0}(x, y), v^{0}(x, y)\right)=\sum_{i=-I}^{I^{\prime}} \sum_{j=-J}^{J^{\prime}}\left(1, u_{i j}, v_{i j}\right) \mathbb{I}_{a_{i j} \leq x \leq b_{i j}} \mathbb{I}_{c_{i j} \leq y \leq d_{i j}}
$$

which is a linear sum of terms as the ones considered in previous sections, we obtain the following properties. As long as there is no collision, Proposition 2.6 gives an approximation of the solution by discrete blocks and when there is a collision in direction $x$ (it is similar in the $y$ direction), Proposition 2.11 gives an approximation of the solution by discrete blocks. Finally, we get the following merging result. 
Proposition 2.12 For any initial data as (2.40), there exists $\left(\rho_{l}, \rho_{l} u_{l}, \rho_{l} v_{l}\right)$ discrete blocks and $\left(\pi_{1}\right)_{l},\left(\pi_{2}\right)_{l} \in \mathcal{M}_{l o c}\left(\left[0, \infty\left[\times \mathbb{R}^{2}\right)\right.\right.$ such that

$$
\left\{\begin{array}{l}
\partial_{t} \rho_{l}+\partial_{x}\left(\rho_{l} u_{l}\right)+\partial_{y}\left(\rho_{l} v_{l}\right)=R_{l} \rightarrow 0, \\
\partial_{t}\left(\rho_{l} u_{l}\right)+\partial_{x}\left(\rho_{l} u_{l}^{2}+\left(\pi_{1}\right)_{l}\right)+\partial_{y}\left(\rho_{l} u_{l} v_{l}\right)=S_{l} \rightarrow 0, \\
\partial_{t}\left(\rho_{l} v_{l}\right)+\partial_{x}\left(\rho_{l} u_{l} v_{l}\right)+\partial_{y}\left(\rho_{l} v_{l}^{2}+\left(\pi_{2}\right)_{l}\right)=T_{l} \rightarrow 0,
\end{array}\right.
$$

in the distribution sense.

Remark 2.3 If we take initial data such that

$$
b_{i+1, j}<a_{i j} \text { and } d_{i, j+1}<c_{i j} \text { for any } i, j,
$$

then collision doesn't appear at time $t=0$ and then we have $\pi_{1}(0, x, y)=0$ and $\pi_{2}(0, x, y)=0$.

We turn now to the proof of $L^{\infty}$ and $B V$ estimates for these functions.

Remark 2.4 Notice that a function $T(z)$ of the form given by Figure 1

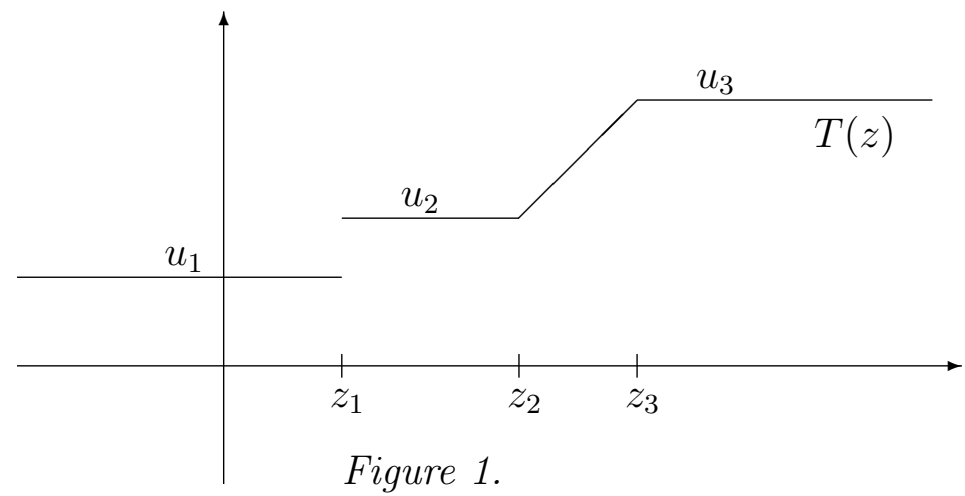

has for derivate a measure in the distributional sense given by

$$
T^{\prime}(z)=\left(u_{2}-u_{1}\right) \delta_{z_{1}}(z)+\frac{u_{3}-u_{2}}{x_{3}-x_{2}} \mathbb{I}_{]_{2}, x_{3}[}(z)
$$

and

$$
\int_{\mathbb{R}}\left|T^{\prime}(z)\right|=\left|u_{2}-u_{1}\right|+\left|u_{3}-u_{2}\right|
$$

Proposition 2.13 For $0 \leq \rho^{0} \leq 1, u^{0}, v^{0} \in L^{\infty}\left(\mathbb{R}^{2}\right) \cap B V\left(\mathbb{R}^{2}\right)$, the blocks of the previous proposition satisfy, for any $t \geq 0$,

$$
\begin{gathered}
0 \leq \rho_{l} \leq 1, \\
\text { essinf } u^{0} \leq u_{l} \leq \text { esssup } u^{0}, \quad \text { essinf } v^{0} \leq v_{l} \leq \text { esssup } v^{0}, \\
\iint_{\mathbb{R}^{2}}\left|\partial_{x} u_{l}(t, x, y)\right| \leq \iint_{\mathbb{R}^{2}}\left|\partial_{x} u^{0}(x, y)\right|, \quad \iint_{\mathbb{R}^{2}}\left|\partial_{y} u_{l}(t, x, y)\right| \leq \iint_{\mathbb{R}^{2}}\left|\partial_{y} u^{0}(x, y)\right|,
\end{gathered}
$$


and

$$
\iint_{\mathbb{R}^{2}}\left|\partial_{x} v_{l}(t, x, y)\right| \leq \iint_{\mathbb{R}^{2}}\left|\partial_{x} v^{0}(x, y)\right|, \quad \iint_{\mathbb{R}^{2}}\left|\partial_{y} v_{l}(t, x, y)\right| \leq \iint_{\mathbb{R}^{2}}\left|\partial_{y} v^{0}(x, y)\right| .
$$

Furthermore, the sequences of measures $\left(\left(\pi_{1}\right)_{l}\right)_{l \geq 1}$ and $\left(\left(\pi_{2}\right)_{l}\right)_{l \geq 1}$ are bounded in $\mathcal{M}_{\text {loc }}\left(\left[0, \infty\left[\times \mathbb{R}^{2}\right)\right.\right.$.

Proof. The $L^{\infty}$ bounds are obvious from construction. For an initial sticky block, using Remark 2.4, we have a relation of the form

$$
\iint_{\mathbb{R}^{2}}\left|\partial_{x} u^{0}(x, y)\right|=\sum_{i=2}^{n^{0}}\left|u_{i}^{0}-u_{i-1}^{0}\right|
$$

We handle the case of a collision in the $x$ direction. Other cases are similar. When a collision happens, for exemple at time $t^{*}$ between blocks $k$ and $k+1$ to simplify the presentation, we have after the collision a speed of the form $u^{*}=u_{k}^{0} c /(c+d)+u_{k+1}^{0} d /(c+d)$ due to Proposition 2.5. Since

$$
\iint_{\mathbb{R}^{2}}\left|\partial_{x} u\left(t^{*}, x, y\right)\right|=\sum_{i=2}^{k-1}\left|u_{i}^{0}-u_{i-1}^{0}\right|+\left|u^{*}-u_{k-1}^{0}\right|+\left|u_{k+2}^{0}-u^{*}\right|+\sum_{i=k+3}^{n^{0}}\left|u_{i}^{0}-u_{i-1}^{0}\right|,
$$

and in order to obtain $\iint_{\mathbb{R}^{2}}\left|\partial_{x} u\left(t^{*}, x, y\right)\right| \leq \iint_{\mathbb{R}^{2}}\left|\partial_{x} u^{0}(x, y)\right|$, we have to prove that

$$
\left|u^{*}-u_{k-1}^{0}\right|+\left|u_{k+2}^{0}-u^{*}\right| \leq\left|u_{k}^{0}-u_{k-1}^{0}\right|+\left|u_{k+1}^{0}-u_{k}^{0}\right|+\left|u_{k+2}^{0}-u_{k+1}^{0}\right| .
$$

First, we have

$$
\begin{aligned}
\left|u_{k+2}^{0}-u^{*}\right| & \leq\left|u_{k+2}^{0}-u_{k+1}^{0}\right|+\left|u_{k+1}^{0}-u^{*}\right| \\
& \leq\left|u_{k+2}^{0}-u_{k+1}^{0}\right|+\left|u_{k+1}^{0} \frac{c+d}{c+d}-u_{k}^{0} \frac{c}{c+d}-u_{k+1}^{0} \frac{d}{c+d}\right| \\
& \leq\left|u_{k+2}^{0}-u_{k+1}^{0}\right|+\frac{c}{c+d}\left|u_{k+1}^{0}-u_{k}^{0}\right| .
\end{aligned}
$$

Similarly, we get $\left|u^{*}-u_{k-1}^{0}\right| \leq\left|u_{k}^{0}-u_{k-1}^{0}\right|+\frac{d}{c+d}\left|u_{k+1}^{0}-u_{k}^{0}\right|$, and by adding these two last inequalities, we get the desired inequality (2.47). Finally, collision after collision, we get the nonincreasing of the quantities of (2.45)-(2.46).

We turn now to the bounds of the measures. Since $\left(\rho_{l}\right)_{l},\left(u_{l}\right)_{l}$ and $\left(v_{l}\right)_{l}$ are $L^{\infty}$ bounded and $S_{l}, T_{l} \rightarrow 0$, we get that $\left(\left(\pi_{1}\right)_{l}\right)_{l}$ and $\left(\left(\pi_{2}\right)_{l}\right)_{l}$ are bounded in the distributional sense. Since they are nonnegative measures, we conclude.

We have now to discretize initial data and to get a stability theorem in order to get solution for the system with constraint for a large class of initial data. 


\section{Discretization and stability}

\section{Discretization with blocks}

We will improve here a result of [2] and [7] in order to get the following approximation lemma of initial data. The difficulty is here to deal with multi-variables functions instead of real-variable functions.

Remark 3.1 The velocities $u$ and $v$ are assumed to be extended linearly in the vacuum $(\rho=0)$ between two successive blocks. Moreover we assume that $u$ and $v$ are constant at $\pm \infty$.

Lemma 3.1 Let $\rho^{0} \in L^{1}\left(\mathbb{R}^{2}\right), u^{0}, v^{0} \in L^{\infty}\left(\mathbb{R}^{2}\right) \cap B V\left(\mathbb{R}^{2}\right)$ such that $0 \leq \rho^{0} \leq 1$. Then, there exists a sequence of block initial data $\left(\rho_{k}^{0}, u_{k}^{0}, v_{k}^{0}\right)_{k \geq 1}$ such that, for any $k \in \mathbb{N}^{*}$,

$$
\rho_{k}^{0} \in L^{1}\left(\mathbb{R}^{2}\right), \quad u_{k}^{0}, v_{k}^{0} \in L^{\infty}\left(\mathbb{R}^{2}\right) \cap B V\left(\mathbb{R}^{2}\right)
$$

with the bounds

$$
\begin{gathered}
0 \leq \rho_{k}^{0} \leq 1, \quad \iint_{\mathbb{R}^{2}} \rho_{k}^{0}(x, y) d x d y \leq \iint_{\mathbb{R}^{2}} \rho^{0}(x, y) d x d y \\
\text { essinf } u^{0} \leq u_{k}^{0} \leq \operatorname{esssup} u^{0}, \quad \text { essinf } v^{0} \leq v_{k}^{0} \leq \operatorname{esssup} v^{0} \\
\iint_{\mathbb{R}^{2}}\left|\partial_{x} u_{k}^{0}(x, y)\right| \leq \iint_{\mathbb{R}^{2}}\left|\partial_{x} u^{0}(x, y)\right|, \quad \iint_{\mathbb{R}^{2}}\left|\partial_{y} u_{k}^{0}(x, y)\right| \leq \iint_{\mathbb{R}^{2}}\left|\partial_{y} u^{0}(x, y)\right|, \\
\iint_{\mathbb{R}^{2}}\left|\partial_{x} v_{k}^{0}(x, y)\right| \leq \iint_{\mathbb{R}^{2}}\left|\partial_{x} v^{0}(x, y)\right|, \quad \iint_{\mathbb{R}^{2}}\left|\partial_{y} v_{k}^{0}(x, y)\right| \leq \iint_{\mathbb{R}^{2}}\left|\partial_{y} v^{0}(x, y)\right|,
\end{gathered}
$$

and for which the convergences $\rho_{k}^{0} \rightarrow \rho^{0}, \rho_{k}^{0} u_{k}^{0} \rightarrow \rho^{0} u^{0}$ and $\rho_{k}^{0} v_{k}^{0} \rightarrow \rho^{0} v^{0}$ hold in the distributional sense.

Proof. Let $k \in \mathbb{N}^{*}$ and set for any $i, j \in \mathbb{Z}$

$$
m_{i j k}=\int_{\frac{i}{k}}^{\frac{i+1}{k}-\frac{1}{k^{2}}} \int_{\frac{j}{k}}^{\frac{j+1}{k}-\frac{1}{k^{2}}} \rho^{0}(x, y) d x d y .
$$

If $m_{i j k} \neq 0$, we set

$$
u_{i j k}^{0}=k^{2} \int_{\frac{i}{k}}^{\frac{i+1}{k}} \int_{\frac{j}{k}}^{\frac{j+1}{k}} u^{0}(x, y) d x d y, \quad v_{i j k}^{0}=k^{2} \int_{\frac{i}{k}}^{\frac{i+1}{k}} \int_{\frac{j}{k}}^{\frac{j+1}{k}} v^{0}(x, y) d x d y .
$$

We finally set, for any $(x, y) \in \mathbb{R}^{2}$,

$$
\begin{aligned}
\rho_{k}^{0}(x, y) & =\sum_{i, j=-k^{2}}^{k^{2}} \mathbb{I}_{\frac{i}{k}, \frac{i}{k}+\sqrt{m_{i j k}}[}(x) \mathbb{I}_{] \frac{j}{k}, \frac{j}{k}+\sqrt{m_{i j k}}}(y), \\
\rho_{k}^{0}(x, y) u_{k}^{0}(x, y) & =\sum_{i, j=-k^{2}}^{k^{2}} u_{i j k}^{0} \mathbb{I}_{] \frac{i}{k}, \frac{i}{k}+\sqrt{m_{i j k}}[}(x) \mathbb{I}_{] \frac{j}{k}, \frac{j}{k}+\sqrt{m_{i j k}}}(y) .
\end{aligned}
$$




$$
\rho_{k}^{0}(x, y) v_{k}^{0}(x, y)=\sum_{i, j=-k^{2}}^{k^{2}} v_{i j k}^{0} \mathbb{I}_{] \frac{i}{k}, \frac{i}{k}+\sqrt{m_{i j k}}[}(x) \mathbb{I}_{\frac{j}{k}, \frac{j}{k}}+\sqrt{m_{i j k}}[(y) .
$$

Notice that $\sqrt{m_{i j k}} \leq \frac{1}{k}-\frac{1}{k^{2}}<\frac{1}{k}$. We have (3.49), in particular since

$$
\begin{aligned}
\iint_{\mathbb{R}^{2}} \rho_{k}^{0}(x, y) d x d y & =\sum_{i, j=-k^{2}}^{k^{2}} m_{i j k} \\
& =\sum_{i, j=-k^{2}}^{k^{2}} \int_{\frac{i}{k}}^{\frac{i+1}{k}-\frac{1}{k^{2}}} \int_{\frac{j}{k}}^{\frac{j+1}{k}-\frac{1}{k^{2}}} \rho^{0}(x, y) d x d y \\
& \leq \iint_{\mathbb{R}^{2}} \rho^{0}(x, y) d x d y
\end{aligned}
$$

We extend the definition of $u_{k}^{0}$ and $v_{k}^{0}$ in the vacuum as in Remark 3.1. We have clearly (3.50). We are considering functions of the form like in Remark 2.4 and then we get that

$$
\int_{\mathbb{R}_{x}}\left|\partial_{x} u_{k}^{0}(x, y)\right|=\sum_{i, j=-k^{2}}^{k^{2}}\left|u_{i j k}^{0}-u_{i-1, j k}^{0}\right| \mathbb{I}_{]} \frac{j}{k}, \frac{j}{k}+\sqrt{m_{i j k}}[(y)
$$

and

$$
\iint_{\mathbb{R}^{2}}\left|\partial_{x} u_{k}^{0}(x, y)\right|=\sum_{i, j=-k^{2}}^{k^{2}}\left|u_{i j k}^{0}-u_{i-1, j k}^{0}\right| \sqrt{m_{i j k}} \leq \sum_{i, j=-k^{2}}^{k^{2}}\left|u_{i j k}^{0}-u_{i-1, j k}^{0}\right| \frac{1}{k} .
$$

Now

$$
\begin{aligned}
\left|u_{i j k}^{0}-u_{i-1, j k}^{0}\right| & =k^{2}\left|\int_{\frac{i}{k}}^{\frac{i+1}{k}} \int_{\frac{j}{k}}^{\frac{j+1}{k}} u^{0}(x, y) d x d y-\int_{\frac{i-1}{k}}^{\frac{i}{k}} \int_{\frac{j}{k}}^{\frac{j+1}{k}} u^{0}(x, y) d x d y\right| \\
& =k^{2}\left|\int_{\frac{i}{k}}^{\frac{i+1}{k}} \int_{\frac{j}{k}}^{\frac{j+1}{k}} u^{0}(x, y)-u^{0}\left(x-\frac{1}{k}, y\right) d x d y\right| \\
& \leq k^{2} \int_{\frac{i}{k}}^{\frac{i+1}{k}} \int_{\frac{j}{k}}^{\frac{j+1}{k}}\left|u^{0}(x, y)-u^{0}\left(x-\frac{1}{k}, y\right)\right| d x d y
\end{aligned}
$$

therefore

$$
\begin{aligned}
\iint_{\mathbb{R}^{2}}\left|\partial_{x} u_{k}^{0}(x, y)\right| & \leq \sum_{i, j=-k^{2}}^{k^{2}} k \int_{\frac{i}{k}}^{\frac{i+1}{k}} \int_{\frac{j}{k}}^{\frac{j+1}{k}}\left|u^{0}(x, y)-u^{0}\left(x-\frac{1}{k}, y\right)\right| d x d y \\
& \leq \iint_{\mathbb{R}^{2}}\left|\frac{u^{0}(x, y)-u^{0}\left(x-\frac{1}{k}, y\right)}{1 / k}\right| d x d y \\
& \leq \iint_{\mathbb{R}^{2}}\left|\partial_{x} u^{0}(x, y)\right|
\end{aligned}
$$


Similarly, we get the other inequalities of (3.51)-(3.52).

Let $\varphi \in C_{c}^{\infty}\left(\mathbb{R}^{2}\right)$ and let $k_{0} \in \mathbb{N}$ such that supp $\varphi \subset\left[-k_{0}, k_{0}\right]^{2}$. Using Taylor formula, there exists $\left.\left(x_{i j k}, y_{i j k}\right) \in\right] \frac{i}{k}, \frac{i}{k}+\sqrt{m_{i j k}}[\times] \frac{j}{k}, \frac{j}{k}+\sqrt{m_{i j k}}[$ such that

$$
\begin{aligned}
& \int_{\frac{i}{k}}^{\frac{i}{k}+\sqrt{m_{i j k}}} \int_{\frac{j}{k}}^{\frac{j}{k}+\sqrt{m_{i j k}}} \varphi(x, y) d x d y \\
= & \varphi\left(\frac{i}{k}, \frac{j}{k}\right) m_{i j k}+\frac{1}{6} \int_{j / k}^{y_{i j k}} \partial_{x x}^{2} \varphi\left(x_{i j k}, v\right) d v m_{i j k}^{3 / 2}+\frac{1}{2} \partial_{x} \varphi\left(x_{i j k}, y_{i j k}\right) m_{i j k}^{3 / 2} \\
& +\frac{1}{2} \partial_{y} \varphi\left(x_{i j k}, y_{i j k}\right) m_{i j k}^{3 / 2}+\frac{1}{6} \int_{i / k}^{x_{i j k}} \partial_{y y}^{2} \varphi\left(u, y_{i j k}\right) d u m_{i j k}^{3 / 2}
\end{aligned}
$$

Now

$$
\iint_{\mathbb{R}^{2}} \rho_{k}^{0}(x, y) \varphi(x, y) d x d y=\sum_{i, j=-k^{2}}^{k^{2}} \int_{\frac{i}{k}}^{\frac{i}{k}+\sqrt{m_{i j k}}} \int_{\frac{j}{k}}^{\frac{j}{k}+\sqrt{m_{i j k}}} \varphi(x, y) d x d y,
$$

and

$$
\varphi\left(\frac{i}{k}, \frac{j}{k}\right) m_{i j k}=\int_{\frac{i}{k}}^{\frac{i+1}{k}-\frac{1}{k^{2}}} \int_{\frac{j}{k}}^{\frac{j+1}{k}-\frac{1}{k^{2}}} \rho^{0}(x, y) \varphi\left(\frac{i}{k}, \frac{j}{k}\right) d x d y,
$$

therefore, for $k>k_{0}$, we have

$$
\begin{aligned}
& \left|\iint_{\mathbb{R}^{2}} \rho_{k}^{0}(x, y) \varphi(x, y) d x d y-\iint_{\mathbb{R}^{2}} \rho^{0}(x, y) \varphi(x, y) d x d y\right| \\
& \leq \sum_{i, j=-k k_{0}}^{k k_{0}-1} \int_{\frac{i}{k}}^{\frac{i+1}{k}-\frac{1}{k^{2}}} \int_{\frac{j}{k}}^{\frac{j+1}{k}-\frac{1}{k^{2}}}\left\|\rho^{0}\right\|_{\infty}\left|\varphi\left(\frac{i}{k}, \frac{j}{k}\right)-\varphi(x, y)\right| d x d y \\
& +\sum_{i, j=-k k_{0}}^{k k_{0}-1}\left(\int_{\frac{i+1}{k}-\frac{1}{k^{2}}}^{\frac{i+1}{k}} \int_{\frac{j}{k}}^{\frac{j+1}{k}}\left\|\rho^{0}\right\|_{\infty}|\varphi(x, y)| d x d y\right. \\
& \left.+\int_{\frac{i}{k}}^{\frac{i+1}{k}-\frac{1}{k^{2}}} \int_{\frac{j+1}{k}-\frac{1}{k^{2}}}^{\frac{j+1}{k}}\left\|\rho^{0}\right\|_{\infty}|\varphi(x, y)| d x d y\right) \\
& +\frac{1}{6} \sum_{i, j=-k k_{0}}^{k k_{0}-1}\left(\left\|\partial_{x x}^{2} \varphi\right\|_{\infty}\left(y_{i j k}-\frac{j}{k}\right)+3\left\|\partial_{x} \varphi\right\|_{\infty}\right. \\
& \left.+3\left\|\partial_{y} \varphi\right\|_{\infty}+\left\|\partial_{y y}^{2} \varphi\right\|_{\infty}\left(x_{i j k}-\frac{i}{k}\right)\right) m_{i j k}^{3 / 2} \\
& \leq \sum_{i, j=-k k_{0}}^{k k_{0}-1} \int_{\frac{i}{k}}^{\frac{i+1}{k}-\frac{1}{k^{2}}} \int_{\frac{j}{k}}^{\frac{j+1}{k}-\frac{1}{k^{2}}}\left(\left\|\partial_{x} \varphi\right\|_{\infty}\left(x-\frac{i}{k}\right)+\left\|\partial_{x} \varphi\right\|_{\infty}\left(y-\frac{j}{k}\right)\right) d x d y \\
& +\|\varphi\|_{\infty} \sum_{i=-k k_{0}}^{k k_{0}-1}\left(\int_{\frac{i+1}{k}-\frac{1}{k^{2}}}^{\frac{i+1}{k}} \int_{\frac{j}{k}}^{\frac{j+1}{k}} d x d y+\int_{\frac{i}{k}}^{\frac{i+1}{k}-\frac{1}{k^{2}}} \int_{\frac{j+1}{k}-\frac{1}{k^{2}}}^{\frac{j+1}{k}} d x d y\right) \\
& +\frac{1}{6} \sum_{i, j=-k k_{0}}^{k k_{0}-1}\left(\left(\left\|\partial_{x x}^{2} \varphi\right\|_{\infty}+\left\|\partial_{y y}^{2} \varphi\right\|_{\infty}\right) \sqrt{m_{i j k}}+3\left(\left\|\partial_{x} \varphi\right\|_{\infty}+\left\|\partial_{y} \varphi\right\|_{\infty}\right)\right)
\end{aligned}
$$




$$
\begin{aligned}
& \times\left(\int_{\frac{i}{k}}^{\frac{i+1}{k}-\frac{1}{k^{2}}} \int_{\frac{j}{k}}^{\frac{j+1}{k}-\frac{1}{k^{2}}} d x d y\right)^{3 / 2} \\
& \leq \sum_{i, j=-k k_{0}}^{k k_{0}-1} \int_{\frac{i}{k}}^{\frac{i+1}{k}-\frac{1}{k^{2}}} \int_{\frac{j}{k}}^{\frac{j+1}{k}-\frac{1}{k^{2}}} d x d y\left(\left\|\partial_{x} \varphi\right\|_{\infty}+\left\|\partial_{x} \varphi\right\|_{\infty}\right) \frac{1}{k} \\
& +\|\varphi\|_{\infty} \sum_{i, j=-k k_{0}}^{k k_{0}-1}\left(\frac{1}{k^{3}}+\frac{1}{k^{4}}\right) \\
& \quad+\frac{1}{6}\left(\left\|\partial_{x x}^{2} \varphi\right\|_{\infty}+\left\|\partial_{y y}^{2} \varphi\right\|_{\infty}+3\left\|\partial_{x} \varphi\right\|_{\infty}+3\left\|\partial_{y} \varphi\right\|_{\infty}\right) \sum_{i, j=-k k_{0}}^{k k_{0}-1}\left(\frac{1}{k^{2}}\right)^{3 / 2} \\
& \leq \quad 4 k^{2} k_{0}^{2} \frac{1}{k^{2}}\left(\left\|\partial_{x} \varphi\right\|_{\infty}+\left\|\partial_{x} \varphi\right\|_{\infty}\right) \frac{1}{k}+\|\varphi\|_{\infty} 4 k^{2} k_{0}^{2}\left(\frac{1}{k^{3}}+\frac{1}{k^{4}}\right) \\
& \quad+\frac{1}{6}\left(\left\|\partial_{x x}^{2} \varphi\right\|_{\infty}+\left\|\partial_{y y}^{2} \varphi\right\|_{\infty}+3\left(\left\|\partial_{x} \varphi\right\|_{\infty}+\left\|\partial_{y} \varphi\right\|_{\infty}\right) 4 k^{2} k_{0}^{2} \frac{1}{k^{3}}\right. \\
& \leq \quad C_{\varphi} \frac{1}{k}
\end{aligned}
$$

and then $\rho_{k}^{0} \rightarrow \rho^{0}$ holds in the distributional sense. We turn now to the convergence of $\rho_{k}^{0} u_{k}^{0}$. For $k>k_{0}$, we have

$$
\begin{gathered}
\left|\iint_{\mathbb{R}^{2}} \rho_{k}^{0}(x, y) u_{k}^{0}(x, y) \varphi(x, y) d x d y-\iint_{\mathbb{R}^{2}} \rho^{0}(x, y) u(x, y) \varphi(x, y) d x d y\right| \\
\leq \sum_{i, j=-k k_{0}}^{k k_{0}-1} \int_{\frac{i}{k}}^{\frac{i+1}{k}-\frac{1}{k^{2}}} \int_{\frac{j}{k}}^{\frac{j+1}{k}-\frac{1}{k^{2}}}\left\|\rho^{0}\right\|_{\infty}\left|u_{i j k}^{0} \varphi\left(\frac{i}{k}, \frac{j}{k}\right)-u^{0}(x, y) \varphi(x, y)\right| d x d y \\
\quad+\sum_{i, j=-k k_{0}}^{k k_{0}-1}\left(\int_{\frac{i+1}{k}-\frac{1}{k^{2}}}^{\frac{i+1}{k}} \int_{\frac{j}{k}}^{\frac{j+1}{k}}\left\|\rho^{0}\right\|_{\infty}\left\|u^{0}\right\|_{\infty}|\varphi(x, y)| d x d y\right. \\
\left.\quad+\int_{\frac{i}{k}}^{\frac{i+1}{k}-\frac{1}{k^{2}}} \int_{\frac{j+1}{k}-\frac{1}{k^{2}}}^{\frac{j+1}{k}}\left\|\rho^{0}\right\|_{\infty}\left\|u^{0}\right\|_{\infty}|\varphi(x, y)| d x d y\right) \\
+\frac{1}{6} u_{i j k}^{0} \sum_{i, j=-k k_{0}}^{k k_{0}-1}\left(\left\|\partial_{x x}^{2} \varphi\right\|_{\infty}\left(y_{i j k}-\frac{j}{k}\right)+3\left\|\partial_{x} \varphi\right\|_{\infty}\right. \\
\left.+3\left\|\partial_{y} \varphi\right\|_{\infty}+\left\|\partial_{y y}^{2} \varphi\right\|_{\infty}\left(x_{i j k}-\frac{i}{k}\right)\right) m_{i j k}^{3 / 2}
\end{gathered}
$$

and the main difference with to regard to the first convergence is the first term. We write

$$
\begin{aligned}
& \sum_{i, j=-k k_{0}}^{k k_{0}-1} \int_{\frac{i}{k}}^{\frac{i+1}{k}-\frac{1}{k^{2}}} \int_{\frac{j}{k}}^{\frac{j+1}{k}-\frac{1}{k^{2}}}\left\|\rho^{0}\right\|_{\infty}\left|u_{i j k}^{0} \varphi\left(\frac{i}{k}, \frac{j}{k}\right)-u^{0}(x, y) \varphi(x, y)\right| d x d y \\
\leq & \sum_{i, j=-k k_{0}}^{k k_{0}-1} \int_{\frac{i}{k}}^{\frac{i+1}{k}-\frac{1}{k^{2}}} \int_{\frac{j}{k}}^{\frac{j+1}{k}-\frac{1}{k^{2}}}\left\|\rho^{0}\right\|_{\infty}\left|u^{0}(x, y)\right|\left|\varphi\left(\frac{i}{k}, \frac{j}{k}\right)-\varphi(x, y)\right| d x d y
\end{aligned}
$$




$$
+\sum_{i, j=-k k_{0}}^{k k_{0}-1} \int_{\frac{i}{k}}^{\frac{i+1}{k}-\frac{1}{k^{2}}} \int_{\frac{j}{k}}^{\frac{j+1}{k}-\frac{1}{k^{2}}}\left\|\rho^{0}\right\|_{\infty}\left|u_{i j k}^{0}-u^{0}(x, y)\right| \varphi\left(\frac{i}{k}, \frac{j}{k}\right) d x d y
$$

and the main new term is in fact the last one. We control it the following way:

$$
\begin{aligned}
& \sum_{i, j=-k k_{0}}^{k k_{0}-1} \int_{\frac{i}{k}}^{\frac{i+1}{k}-\frac{1}{k^{2}}} \int_{\frac{j}{k}}^{\frac{j+1}{k}-\frac{1}{k^{2}}}\left\|\rho^{0}\right\|_{\infty}\left|u_{i j k}^{0}-u^{0}(x, y)\right| \varphi\left(\frac{i}{k}, \frac{j}{k}\right) d x d y \\
\leq & \sum_{i, j=-k k_{0}}^{k k_{0}-1} \int_{\frac{i}{k}}^{\frac{i+1}{k}-\frac{1}{k^{2}}} \int_{\frac{j}{k}}^{\frac{j+1}{k}-\frac{1}{k^{2}}}\left\|\rho^{0}\right\|_{\infty}\left|\Delta_{i j k}^{u^{0}}(x, y)\right|\|\varphi\|_{\infty} d x d y,
\end{aligned}
$$

where

$$
\Delta_{i j k}^{u^{0}}(x, y)=k^{2} \int_{\frac{i}{k}}^{\frac{i+1}{k}} \int_{\frac{j}{k}}^{\frac{j+1}{k}}\left(u^{0}(\tilde{x}, \tilde{y})-u^{0}(x, y)\right) d \tilde{x} d \tilde{y}
$$

Now $u^{0} \in B V\left(\mathbb{R}^{2}\right)$, then $u^{0}$ is continuous and then uniformly continuous on compacts. Let $\varepsilon>0$, there exists $\eta>0$ such that for any $(x, y),(\tilde{x}, \tilde{y}) \in$ $\left[-k_{0}, k_{0}\right]^{2}$, if $|x-\tilde{x}| \leq \eta,|y-\tilde{y}| \leq \eta$, then $\left|u^{0}(\tilde{x}, \tilde{y})-u^{0}(x, y)\right| \leq \varepsilon$. Now for $i, j \in \mathbb{Z} \cap\left[-k k_{0}, k k_{0}-1\right]$ and $x, \tilde{x} \in\left[\frac{i}{k}, \frac{i+1}{k}\right], y, \tilde{y} \in\left[\frac{j}{k}, \frac{j+1}{k}\right]$, then $(x, y),(\tilde{x}, \tilde{y}) \in\left[-k_{0}, k_{0}\right]^{2}$. Thus for $\frac{1}{k}<\eta$, we have

$$
\begin{aligned}
& \sum_{i, j=-k k_{0}}^{k k_{0}-1} \int_{\frac{i}{k}}^{\frac{i+1}{k}-\frac{1}{k^{2}}} \int_{\frac{j}{k}}^{\frac{j+1}{k}-\frac{1}{k^{2}}}\left\|\rho^{0}\right\|_{\infty}\left|u_{i j k}^{0}-u^{0}(x, y)\right| \varphi\left(\frac{i}{k}, \frac{j}{k}\right) d x d y \\
\leq & \sum_{i, j=-k k_{0}}^{k k_{0}-1} \int_{\frac{i}{k}}^{\frac{i+1}{k}-\frac{1}{k^{2}}} \int_{\frac{j}{k}}^{\frac{j+1}{k}-\frac{1}{k^{2}}}\left\|\rho^{0}\right\|_{\infty}\left|k^{2} \int_{\frac{i}{k}}^{\frac{i+1}{k}} \int_{\frac{j}{k}}^{\frac{j+1}{k}} \varepsilon d \tilde{x} d \tilde{y}\right|\|\varphi\|_{\infty} d x d y \\
\leq & \varepsilon \sum_{i, j=-k k_{0}}^{k k_{0}-1} \frac{1}{k^{2}}=4 k_{0}^{2} \varepsilon .
\end{aligned}
$$

It gives the limit of the new term and we get that $\rho_{k}^{0} u_{k}^{0} \rightarrow \rho^{0} u^{0}$ holds in the distributional sense. Similarly we obtain the convergence of $\rho_{k}^{0} v_{k}^{0}$.

Remark 3.2 Notice that we have $\sqrt{m_{i j k}} \leq \frac{1}{k}-\frac{1}{k^{2}}<\frac{1}{k}$ and then we are in the situation of (2.42) in Remark 3.53 and thus this discretization by blocks will lead to solutions with no initial measure.

\section{Stability Theorem}

The results we prove in this section have two specific purposes. First, we prove stability of solutions. Secondly, we prove the existence of more solutions than those obtained in previous sections by passing to the limit in some particular solutions.

But let's start with a few technical results. The first one is to help us passing to the limit in the products. It is an extension in dimension two of a similar Lemma in dimension one proved in [2]. 
Lemma 3.2 Consider for any $k \in \mathbb{N}$, some functions $\gamma_{k} \in L^{\infty}(] 0, T\left[\times \mathbb{R}^{2}\right)$, $\omega_{k} \in L^{\infty}(] 0, T\left[, B V\left(\mathbb{R}^{2}\right)\right)$ and $\gamma \in L^{\infty}(] 0, T\left[\times \mathbb{R}^{2}\right), \omega \in L^{\infty}(] 0, T\left[, B V\left(\mathbb{R}^{2}\right)\right)$. Let us assume that $\left(\gamma_{k}\right)_{k \in \mathbb{N}}$ is a bounded sequence in $L^{\infty}\left(10, T\left[\times \mathbb{R}^{2}\right)\right.$ that tends to $\gamma$ in $L_{w *}^{\infty}(] 0, T\left[\times \mathbb{R}^{2}\right)$, and satisfies, for any $\Gamma \in C_{c}^{\infty}\left(\mathbb{R}^{2}\right)$,

$$
\iint_{\mathbb{R}^{2}}\left(\gamma_{k}-\gamma\right)(t, x, y) \Gamma(x, y) d x d y \underset{k \rightarrow+\infty}{\rightarrow} 0
$$

either $i)$ a.e. $t \in] 0, T[$ or $i i)$ in $L_{t}^{1}(] 0, T[)$. Let us also assume that $\left(\omega_{k}\right)_{k \in \mathbb{N}}$ is a bounded sequence in $L^{\infty}(] 0, T\left[\times \mathbb{R}^{2}\right)$ that tends to $\omega$ in $L_{w *}^{\infty}(] 0, T\left[\times \mathbb{R}^{2}\right)$, and such that, for any $t \in[0, T]$,

$$
\begin{array}{cc}
\iint_{\mathbb{R}^{2}}\left|\partial_{x} \omega_{k}(t, x, y)\right| \leq C, & \iint_{\mathbb{R}^{2}}\left|\partial_{y} \omega_{k}(t, x, y)\right| \leq C, \\
\iint_{\mathbb{R}^{2}}\left|\partial_{x} \omega(t, x, y)\right| \leq C, & \iint_{\mathbb{R}^{2}}\left|\partial_{y} \omega(t, x, y)\right| \leq C,
\end{array}
$$

with $C$ being a constant. Then $\gamma_{k} \omega_{k} \rightarrow \gamma \omega$ in $L_{w *}^{\infty}(] 0, T\left[\times \mathbb{R}^{2}\right)$, as $k \rightarrow+\infty$.

Proof. We detail case $i$ ), the proof being very similar for case $i i$ ). Let $\zeta_{\varepsilon}$ be a sequence of mollifiers in $\mathbb{R}^{2}$. We shall use the notation $z^{\varepsilon}=z x_{x y}^{*} \zeta_{\varepsilon}$. Let us write the decomposition

$$
\gamma_{k} \omega_{k}-\gamma \omega=\gamma_{k}\left(\omega_{k}-\omega_{k}^{\varepsilon}\right)+\left(\gamma_{k}-\gamma\right) \omega_{k}^{\varepsilon}+\gamma\left(\omega_{k}^{\varepsilon}-\omega^{\varepsilon}\right)+\gamma\left(\omega^{\varepsilon}-\omega\right) .
$$

We are first going to control the first and fourth terms of this decomposition for $\varepsilon$ small enough and uniformly in $k$. Then, fixing $\varepsilon$, we shall pass to the limit, when $k$ tends towards infinity, in the second and third terms. Let $\varphi \in$ $C_{c}^{\infty}(] 0, T\left[\times \mathbb{R}^{2}\right)$.

- Let $\eta>0$. The term $\int_{0}^{T} \iint_{\mathbb{R}^{2}} \gamma\left(\omega^{\varepsilon}-\omega\right) \varphi d x d y d t$ is controlled in the following way. Since $\omega \in L^{\infty}(] 0, T\left[, B V\left(\mathbb{R}^{2}\right)\right)$, we have

$$
\iint_{\mathbb{R}^{2}}|\omega(t, x-\tilde{x}, y-\tilde{y})-\omega(t, x, y)| d x d y \leq|\tilde{x}| \iint_{\mathbb{R}^{2}}\left|\partial_{x} \omega(t, ., .)\right|+|\tilde{y}| \iint_{\mathbb{R}^{2}}\left|\partial_{y} \omega(t, ., .)\right|,
$$

hence for $\varepsilon<1$,

$$
\begin{aligned}
& \left\|\omega^{\varepsilon}(t, ., .)-\omega(t, ., .)\right\|_{L^{1}\left(\mathbb{R}^{2}\right)} \\
= & \iint_{\mathbb{R}^{2}}\left|\iint_{\mathbb{R}^{2}}(\omega(t, x-\tilde{x}, y-\tilde{y})-\omega(t, x, y)) \zeta_{\varepsilon}(y) d \tilde{x} d \tilde{y}\right| d x d y \\
\leq & C \int_{B(0, \varepsilon)}(|\tilde{x}|+|\tilde{y}|) \zeta_{\varepsilon}(\tilde{x}, \tilde{y}) d \tilde{x} d \tilde{y} \leq \varepsilon \tilde{C}
\end{aligned}
$$

where $\tilde{C}$ is a constant independent of $\varepsilon$ and $t$. Thus we get

$$
\left|\int_{0}^{T} \iint_{\mathbb{R}^{2}} \gamma\left(\omega^{\varepsilon}-\omega\right) \varphi d x d y d t\right| \leq \varepsilon \tilde{C}\|\varphi\|_{\infty}\|\gamma\|_{\infty}
$$


This is less than $\eta$ if $\varepsilon$ is small enough. We have the same bound uniformly in $k$ for $\left(\gamma_{k}\right)_{k \geq 0}$ and $\left(\omega_{k}\right)_{k \geq 0}$, thus for such $\varepsilon$,

$$
\left|\int_{0}^{T} \iint_{\mathbb{R}^{2}} \gamma\left(\omega^{\varepsilon}-\omega\right) \varphi d x d y d t\right| \leq \eta \text { and }\left|\int_{0}^{T} \iint_{\mathbb{R}^{2}} \gamma_{k}\left(\omega_{k}^{\varepsilon}-\omega_{k}\right) \varphi d x d y d t\right| \leq \eta, \quad \forall k \in \mathbb{N}
$$

- Let now $\varepsilon$ be fixed as above. For the third term of the decomposition (3.59), obviously $\omega_{k}^{\varepsilon}-\omega^{\varepsilon} \rightarrow 0$ in $L_{w *}^{\infty}(] 0, T\left[\times \mathbb{R}^{2}\right)$, thus $\gamma\left(\omega_{k}^{\varepsilon}-\omega^{\varepsilon}\right) \rightarrow 0$.

It remains to establish the convergence $\left(\gamma_{k}-\gamma\right) \omega_{k}^{\varepsilon} \rightarrow 0$ in $L_{w *}^{\infty}(] 0, T\left[\times \mathbb{R}^{2}\right)$. In order to do this, we only need to consider a test function $\varphi \in C_{c}^{\infty}(] 0, T\left[\times \mathbb{R}^{2}\right)$, $\varphi(t, x, y)=\varphi_{1}(t) \varphi_{2}(x, y), \varphi_{1} \in C_{c}^{\infty}(] 0, T[), \varphi_{2} \in C_{c}^{\infty}\left(\mathbb{R}^{2}\right)$. In order to prove that

$$
\int_{0}^{T} \iint_{\mathbb{R}^{2}}\left(\gamma_{k}-\gamma\right)(t, x, y) \omega_{k}^{\varepsilon}(t, x, y) \varphi(t, x, y) d x d y d t \rightarrow 0, \quad k \rightarrow \infty
$$

we write this integral as $\int_{0}^{T} \iint_{\mathbb{R}^{2}} I_{k}(t, \tilde{x}, \tilde{y}) d \tilde{x} d \tilde{y} d t$ where

$$
I_{k}(t, \tilde{x}, \tilde{y})=\omega_{k}(t, \tilde{x}, \tilde{y})\left(\iint_{\mathbb{R}^{2}}\left(\gamma_{k}-\gamma\right)(t, x, y) \zeta_{\varepsilon}(x-\tilde{x}, y-\tilde{y}) \varphi(t, x, y) d x d y\right)
$$

We are going to prove the convergence of this integral using Lebesgue's theorem. Since, at $(\tilde{x}, \tilde{y})$ being fixed, $(x, y) \mapsto \zeta_{\varepsilon}(x-\tilde{x}, y-\tilde{y}) \varphi_{2}(x, y) \in C_{c}^{\infty}\left(\mathbb{R}^{2}\right)$ and together with the fact that $\left(\omega_{k}\right)_{k \geq 0}$ is bounded in $L^{\infty}(] 0, T\left[\times \mathbb{R}^{2}\right)$, we deduce from the property of $\gamma$, that for a.e. $t, x, y, I_{k}(t, x, y) \rightarrow 0$ as $k \rightarrow \infty$. We also have the following estimate,

$$
\left|I_{k}(t, \tilde{x}, \tilde{y})\right| \leq \sup _{k}\left\|\omega_{k}\right\|_{L^{\infty}}\left(\sup _{k}\left\|\gamma_{k}\right\|_{L^{\infty}}+\|\gamma\|_{L^{\infty}}\right) J(t, \tilde{x}, \tilde{y}),
$$

where $J:(t, \tilde{x}, \tilde{y}) \mapsto \iint_{\mathbb{R}^{2}} \zeta_{\varepsilon}(x-\tilde{x}, y-\tilde{y})|\varphi(t, x, y)| d x d y \in L^{1}(] 0, T\left[\times \mathbb{R}^{2}\right)$. Therefore, by dominated convergence, we have that $I_{k}(t, x, y) \rightarrow 0$ in $L^{1}(] 0, T\left[\times \mathbb{R}^{2}\right)$, which gives the desired convergence.

- Finally, we can conclude that $\gamma_{n} \omega_{n}-\gamma \omega \rightarrow 0$ in $L_{w *}^{\infty}(] 0, T[\times \mathbb{R})$.

Remark 3.3 This is a result of compensated compactness, which uses the compactness in $(x, y)$ for $\left(\omega_{k}\right)_{k}$ given by (3.57) and the weak compactness in $t$ for $\left(\gamma_{k}\right)_{k}$ given by (3.56) to pass to the weak limit in the product $\gamma_{k} \omega_{k}$.

The second result gives some continuity in time. The proof is an easy adaptation in dimension two of Lemma 4.4 of [5]. The main idea is to use a countable dense set in $C_{c}^{\infty}\left(\mathbb{R}^{2}\right)$ for the $L^{1}$-norm and Ascoli's Theorem. Since there is no new difficulty, we skip the proof. 
Lemma 3.3 Let $\left(n_{k}\right)_{k \in \mathbb{N}^{*}}$ be a bounded sequence in $L^{\infty}(] 0, T\left[\times \mathbb{R}^{2}\right)$ which satisfies:

for all $\varphi \in C_{c}^{\infty}\left(\mathbb{R}^{2}\right)$, the sequence $\left(\int_{\mathbb{R}} n_{k}(t, x, y) \varphi(x, y) d x d y\right)_{k}$ is uniformly Lipschitz continuous on $[0, T]$, i.e. $\exists C_{\varphi}>0, \forall k \in \mathbb{N}^{*}, \forall s, t \in[0, T]$,

$$
\left|\iint_{\mathbb{R}^{2}}\left(n_{k}(t, x, y)-n_{k}(s, x, y)\right) \varphi(x, y) d x d y\right| \leq C_{\varphi}|t-s| .
$$

Then, up to a subsequence, it exists $n \in L^{\infty}(] 0, T\left[\times \mathbb{R}^{2}\right)$ such that $n_{k} \rightarrow n$ in $C\left([0, T], L_{w *}^{\infty}\left(\mathbb{R}^{2}\right)\right)$, i.e.

$$
\forall \Gamma \in L^{1}\left(\mathbb{R}^{2}\right), \quad \sup _{t \in[0, T]}\left|\iint_{\mathbb{R}^{2}}\left(n_{k}(t, x, y)-n(t, x, y)\right) \Gamma(x, y) d x d y\right| \underset{k \rightarrow+\infty}{\rightarrow} 0 .
$$

We prove now the stability result.

Theorem 3.4 (Stability of solutions) Let us consider a sequence of solutions $\left(\rho_{k}, u_{k}, v_{k},\left(\pi_{1}\right)_{k},\left(\pi_{2}\right)_{k}\right)_{k \geq 1}$, with regularities (1.8)-(1.10), satisfying (1.4) with the constraints (1.5)-(1.6) and initial data $\left(\rho_{k}^{0}, u_{k}^{0}, v_{k}^{0}\right)$. We assume the following bounds for initial data:

$$
\begin{gathered}
\left(\rho_{k}^{0}\right)_{k \geq 1} \text { is bounded in } L^{\infty}\left(\mathbb{R}^{2}\right) \text { and in } L^{1}\left(\mathbb{R}^{2}\right), \\
\left(u_{k}^{0}\right)_{k \geq 1},\left(v_{k}^{0}\right)_{k \geq 1} \text { are bounded in } L^{\infty}\left(\mathbb{R}^{2}\right) \text { and in } B V\left(\mathbb{R}^{2}\right) .
\end{gathered}
$$

The solutions are supposed to satisfy

$$
\begin{gathered}
0 \leq \rho_{k} \leq 1, \quad \iint_{\mathbb{R}^{2}} \rho_{k}(t, x, y) d x d y \leq \iint_{\mathbb{R}^{2}} \rho_{k}^{0}(x, y) d x d y \\
\text { essinf } u_{k}^{0} \leq u_{k} \leq \operatorname{esssup} u_{k}^{0}, \quad \text { essinf } v_{k}^{0} \leq v_{k} \leq \text { esssup } v_{k}^{0}, \\
\iint_{\mathbb{R}^{2}}\left|\partial_{x} u_{k}(t, x, y)\right| \leq \iint_{\mathbb{R}^{2}}\left|\partial_{x} u_{k}^{0}(x, y)\right|, \quad \iint_{\mathbb{R}^{2}}\left|\partial_{y} u_{k}(t, x, y)\right| \leq \iint_{\mathbb{R}^{2}}\left|\partial_{y} u_{k}^{0}(x, y)\right|, \\
\iint_{\mathbb{R}^{2}}\left|\partial_{x} v_{k}(t, x, y)\right| \leq \iint_{\mathbb{R}^{2}}\left|\partial_{x} v_{k}^{0}(x, y)\right|, \quad \iint_{\mathbb{R}^{2}}\left|\partial_{y} v_{k}(t, x, y)\right| \leq \iint_{\mathbb{R}^{2}}\left|\partial_{y} v_{k}^{0}(x, y)\right|,
\end{gathered}
$$

and finally we assume that

$$
\left(\left(\pi_{1}\right)_{k}\right)_{k \geq 1} \text { and }\left(\left(\pi_{2}\right)_{k}\right)_{k \geq 1} \text { are bounded in } \mathcal{M}_{l o c}\left(\left[0, \infty\left[\times \mathbb{R}^{2}\right)\right.\right. \text {. }
$$

Then, extracting a subsequence if necessary, as $k \rightarrow \infty$, we have in the distributional sense $\left(\rho_{k}, u_{k}, v_{k},\left(\pi_{1}\right)_{k},\left(\pi_{2}\right)_{k}\right) \rightarrow\left(\rho, u, v, \pi_{1}, \pi_{2}\right)$, where $\left(\rho, u, v, \pi_{1}, \pi_{2}\right)$, with regularities (1.8)-(1.10), are solution of (1.4) with the constraints (1.5) and satisfy the bounds

$$
0 \leq \rho \leq 1, \quad \iint_{\mathbb{R}^{2}} \rho(t, x, y) d x d y \leq \iint_{\mathbb{R}^{2}} \rho^{0}(x, y) d x d y
$$




$$
\begin{gathered}
\text { essinf } u^{0} \leq u \leq \text { esssup } u^{0}, \quad \text { essinf } v^{0} \leq v \leq e \operatorname{essup} v^{0} \\
\iint_{\mathbb{R}^{2}}\left|\partial_{x} u(t, x, y)\right| \leq \iint_{\mathbb{R}^{2}}\left|\partial_{x} u^{0}(x, y)\right|, \quad \iint_{\mathbb{R}^{2}}\left|\partial_{y} u(t, x, y)\right| \leq \iint_{\mathbb{R}^{2}}\left|\partial_{y} u^{0}(x, y)\right| \\
\iint_{\mathbb{R}^{2}}\left|\partial_{x} v(t, x, y)\right| \leq \iint_{\mathbb{R}^{2}}\left|\partial_{x} v^{0}(x, y)\right|, \quad \iint_{\mathbb{R}^{2}}\left|\partial_{y} v(t, x, y)\right| \leq \iint_{\mathbb{R}^{2}}\left|\partial_{y} v^{0}(x, y)\right|
\end{gathered}
$$

Furthermore we get the existence of measures $\tilde{\pi}_{1}$ and $\tilde{\pi}_{2} \in \mathcal{M}_{\text {loc }}\left(\left[0, \infty\left[\times \mathbb{R}^{2}\right)\right.\right.$ such that

$$
\pi_{1}=\tilde{\pi}_{1}, \quad \pi_{2}=\tilde{\pi}_{2},
$$

which is a weak formulation for (1.6).

Proof. Since $\left(\rho_{k}, u_{k}, v_{k}\right)_{k \geq 1}$ are bounded in $L^{\infty}(] 0,+\infty\left[\times \mathbb{R}^{2}\right)$, then there exists a subsequence such that

$$
\rho_{k} \rightarrow \rho, u_{k} \rightarrow u, v_{k} \rightarrow v \text { in } L_{w *}^{\infty}(] 0,+\infty\left[\times \mathbb{R}^{2}\right) .
$$

From (3.66), there exists a subsequence such that

$$
\left(\pi_{1}\right)_{k} \rightarrow \pi_{1},\left(\pi_{2}\right)_{k} \rightarrow \pi_{2} \text { in } \mathcal{M}_{l o c}\left(\left[0, \infty\left[\times \mathbb{R}^{2}\right) .\right.\right.
$$

From the first equation of (1.4), the sequence $\left(\rho_{k}\right)_{k \geq 1}$ satifies the estimate: $\forall T>0, \forall \varphi \in C_{c}^{\infty}\left(\mathbb{R}^{2}\right), \forall t, s \in[0, T], \forall k \in \mathbb{N}^{*}$,

$$
\left|\iint_{\mathbb{R}^{2}}\left(\rho_{k}(t, x, y)-\rho_{k}(s, x, y)\right) \varphi(x, y) d x d y\right| \leq C_{\varphi}|t-s|,
$$

with

$$
C_{\varphi}=\sup _{k \geq 1}\left\|u_{k}^{0}\right\|_{L^{\infty}}\left(\iint_{\mathbb{R}^{2}}\left|\partial_{x} \varphi\right| d x d y\right)+\sup _{k \geq 1}\left\|v_{k}^{0}\right\|_{L^{\infty}}\left(\iint_{\mathbb{R}^{2}}\left|\partial_{y} \varphi\right| d x d y\right) .
$$

Then, applying Lemma 3.3, $\rho_{k} \rightarrow \rho$ in $C\left([0, T], L_{w *}^{\infty}\left(\mathbb{R}^{2}\right)\right)$. Furthermore $\left(u_{k}\right)_{k \geq 1}$ is bounded in $B V\left(\mathbb{R}^{2}\right)$ uniformly in time thanks to (3.61) and (3.64). We can then apply Lemma 3.2 and we get that $\rho_{k} u_{k} \rightarrow \rho u$ in $L_{w *}^{\infty}(] 0, T\left[\times \mathbb{R}^{2}\right)$. Similarly, we have $\rho_{k} v_{k} \rightarrow \rho v$ in $L_{w *}^{\infty}(] 0, T\left[\times \mathbb{R}^{2}\right)$.

Now the second equation of (1.4) gives that

$$
\begin{aligned}
& \frac{d}{d t} \iint_{\mathbb{R}^{2}}\left(\rho_{k} u_{k}\right)(t, x, y) \varphi(x, y) d x d y \\
= & \iint_{\mathbb{R}^{2}}\left(\rho_{k} u_{k}^{2}\right)(t, x, y) \partial_{x} \varphi(x, y) d x d y+\iint_{\mathbb{R}^{2}}\left(\rho_{k} u_{k} v_{k}\right)(t, x, y) \partial_{y} \varphi(x, y) d x d y \\
& +\iint_{\mathbb{R}^{2}} \partial_{x} \varphi(x, y)\left(\pi_{1}\right)_{k}(t, x, y),
\end{aligned}
$$


thus the sequence $\iint_{\mathbb{R}^{2}}\left(\rho_{k} u_{k}\right)(t, x, y) \varphi(x, y) d x d y$ is bounded in $B V_{t}$. Therefore, in the same spirit than the proof of Lemma 3.3 (see also [5]), we can extract a subsequence such that

$$
\iint_{\mathbb{R}^{2}}\left(\rho_{k} u_{k}\right)(t, x, y) \varphi(x, y) d x d y \rightarrow \iint_{\mathbb{R}^{2}}(\rho u)(t, x, y) \varphi(x, y) d x d y \text { in } L^{1}(] 0, T[),
$$

for all $\varphi \in C_{c}^{\infty}\left(\mathbb{R}^{2}\right)$. We can then apply Lemma 3.2 with $\gamma_{k}=\rho_{k} u_{k}$ this time and $\omega_{k}=u_{k}$ (and also with $v_{k}$ ) and we get that $\rho_{k} u_{k}^{2} \rightarrow \rho u^{2}$ and $\rho_{k} u_{k} v_{k} \rightarrow \rho u v$ in $L_{w *}^{\infty}(] 0, T\left[\times \mathbb{R}^{2}\right)$. Similarly, we also have $\rho_{k} v_{k}^{2} \rightarrow \rho v^{2}$ in $L_{w *}^{\infty}\left(10, T\left[\times \mathbb{R}^{2}\right)\right.$. We can now pass to the limit in the weak formulation to get (1.11)-(1.13) with the initial data $\left(\rho^{0}, u^{0}, v^{0}\right)$. Finally, since $\left(\rho_{k}\left(\pi_{1}\right)_{k}\right)_{k}$ and $\left(\rho_{k}\left(\pi_{2}\right)_{k}\right)_{k}$ are bounded in the measure, up to a subsequence, we have the existence of $\tilde{\pi}_{1}, \tilde{\pi}_{2} \in$ $\mathcal{M}_{\text {loc }}\left(\left[0, \infty\left[\times \mathbb{R}^{2}\right)\right.\right.$ such that

$$
\rho_{k}\left(\pi_{1}\right)_{k} \rightarrow \tilde{\pi}_{1}, \rho_{k}\left(\pi_{2}\right)_{k} \rightarrow \tilde{\pi}_{2} \text { in } \mathcal{M}_{l o c}\left(\left[0, \infty\left[\times \mathbb{R}^{2}\right) .\right.\right.
$$

Since $\rho_{k}\left(\pi_{1}\right)_{k}=\left(\pi_{1}\right)_{k}$ and $\rho_{k}\left(\pi_{2}\right)_{k}=\left(\pi_{2}\right)_{k}$, at the limit, we get (3.71).

Remark 3.4 The relation (3.71) is a weak formulation of (1.6). We will come back in the last section to more relations between both formulations.

Remark 3.5 We have the same stability result assuming (3.71) instead of (1.6) in the assumptions of the theorem.

\section{Limit of approximate solutions}

In dimension one, we directly obtained explicite solutions for any block initial data. In the present dimension-two case, at this step, we only have approximation of solution for general block initial data. We need to improve the previous stability result in the case where we only have

$$
\left\{\begin{array}{l}
\partial_{t} \rho_{l}+\partial_{x}\left(\rho_{l} u_{l}\right)+\partial_{y}\left(\rho_{l} v_{l}\right)=R_{l} \rightarrow 0 \\
\partial_{t}\left(\rho_{l} u_{l}\right)+\partial_{x}\left(\rho_{l} u_{l}^{2}+\left(\pi_{1}\right)_{l}\right)+\partial_{y}\left(\rho_{l} u_{l} v_{l}\right)=S_{l} \rightarrow 0 \\
\partial_{t}\left(\rho_{l} v_{l}\right)+\partial_{x}\left(\rho_{l} u_{l} v_{l}\right)+\partial_{y}\left(\rho_{l} v_{l}^{2}+\left(\pi_{2}\right)_{l}\right)=T_{l} \rightarrow 0
\end{array}\right.
$$

instead of having $R_{l}=S_{l}=T_{l}=0$. We prove now that in this situation, we can extract a subsequence whose limit is a solution.

Theorem 3.5 (Limit of approximations) Let us consider a sequence $\left(\rho_{l}, u_{l}\right.$, $\left.v_{l},\left(\pi_{1}\right)_{l},\left(\pi_{2}\right)_{l}\right)_{l \geq 1}$, with regularities (1.8)-(1.10), satisfying (3.76) with the constraints (1.5)-(1.6) and initial data $\left(\rho_{l}^{0}, u_{l}^{0}, v_{l}^{0}\right)$. We assume the following bounds for initial data:

$$
\begin{gathered}
\left(\rho_{l}^{0}\right)_{l \geq 1} \text { is bounded in } L^{\infty}\left(\mathbb{R}^{2}\right) \text { and in } L^{1}\left(\mathbb{R}^{2}\right), \\
\left(u_{l}^{0}\right)_{l \geq 1},\left(v_{l}^{0}\right)_{l \geq 1} \text { are bounded in } L^{\infty}\left(\mathbb{R}^{2}\right) \text { and in } B V\left(\mathbb{R}^{2}\right) .
\end{gathered}
$$


The functions are supposed to satisfy the bounds

$$
\begin{gathered}
0 \leq \rho_{l} \leq 1, \quad \iint_{\mathbb{R}^{2}} \rho_{l}(t, x, y) d x d y \leq \iint_{\mathbb{R}^{2}} \rho_{l}^{0}(x, y) d x d y \\
\text { essinf } u_{l}^{0} \leq u_{l} \leq \text { esssup } u_{l}^{0}, \quad \text { essinf } v_{l}^{0} \leq v_{l} \leq \text { esssup } v_{l}^{0}, \\
\iint_{\mathbb{R}^{2}}\left|\partial_{x} u_{l}(t, x, y)\right| \leq \iint_{\mathbb{R}^{2}}\left|\partial_{x} u_{l}^{0}(x, y)\right|, \quad \iint_{\mathbb{R}^{2}}\left|\partial_{y} u_{l}(t, x, y)\right| \leq \iint_{\mathbb{R}^{2}}\left|\partial_{y} u_{l}^{0}(x, y)\right|, \\
\iint_{\mathbb{R}^{2}}\left|\partial_{x} v_{l}(t, x, y)\right| \leq \iint_{\mathbb{R}^{2}}\left|\partial_{x} v_{l}^{0}(x, y)\right|, \quad \iint_{\mathbb{R}^{2}}\left|\partial_{y} v_{l}(t, x, y)\right| \leq \iint_{\mathbb{R}^{2}}\left|\partial_{y} v_{l}^{0}(x, y)\right|,
\end{gathered}
$$

and finally we assume that

$$
\left(\left(\pi_{1}\right)_{l}\right)_{l \geq 1} \text { and }\left(\left(\pi_{2}\right)_{l}\right)_{l \geq 1} \text { are bounded in } \mathcal{M}_{l o c}\left(\left[0, \infty\left[\times \mathbb{R}^{2}\right)\right.\right. \text {. }
$$

Then, extracting a subsequence if necessary, as $l \rightarrow \infty$, we have in the distributional sense $\left(\rho_{l}, u_{l}, v_{l},\left(\pi_{1}\right)_{l},\left(\pi_{2}\right)_{l}\right) \rightarrow\left(\rho, u, v, \pi_{1}, \pi_{2}\right)$, where $\left(\rho, u, v, \pi_{1}, \pi_{2}\right)$ have regularities (1.8)-(1.10), are solution of (1.4) with the constraints (1.5) and satisfy the bounds

$$
\begin{gathered}
0 \leq \rho \leq 1, \quad \iint_{\mathbb{R}^{2}} \rho(t, x, y) d x d y \leq \iint_{\mathbb{R}^{2}} \rho^{0}(x, y) d x d y \\
\operatorname{essinf} u^{0} \leq u \leq \operatorname{esssup} u^{0}, \quad \text { essinf } v^{0} \leq v \leq \operatorname{esssup} v^{0} \\
\iint_{\mathbb{R}^{2}}\left|\partial_{x} u(t, x, y)\right| \leq \iint_{\mathbb{R}^{2}}\left|\partial_{x} u^{0}(x, y)\right|, \quad \iint_{\mathbb{R}^{2}}\left|\partial_{y} u(t, x, y)\right| \leq \iint_{\mathbb{R}^{2}}\left|\partial_{y} u^{0}(x, y)\right| \\
\iint_{\mathbb{R}^{2}}\left|\partial_{x} v(t, x, y)\right| \leq \iint_{\mathbb{R}^{2}}\left|\partial_{x} v^{0}(x, y)\right|, \quad \iint_{\mathbb{R}^{2}}\left|\partial_{y} v(t, x, y)\right| \leq \iint_{\mathbb{R}^{2}}\left|\partial_{y} v^{0}(x, y)\right|
\end{gathered}
$$

Furthermore we get the existence of measures $\tilde{\pi}_{1}$ and $\tilde{\pi}_{2} \in \mathcal{M}_{l o c}\left(\left[0, \infty\left[\times \mathbb{R}^{2}\right)\right.\right.$ such that

$$
\pi_{1}=\tilde{\pi}_{1}, \quad \pi_{2}=\tilde{\pi}_{2},
$$

which is a weak formulation for (1.6).

Proof. The proof is very similar to the one of Thereom 3.4 except an important difference, which is the relation (3.74). Here we get a relation of the form

$$
\left|\iint_{\mathbb{R}^{2}}\left(\rho_{k}(t, x, y)-\rho_{k}(s, x, y)\right) \varphi(x, y) d x d y\right| \leq C_{\varphi}|t-s|+\left|\int_{s}^{t} \iint_{\mathbb{R}^{2}} R_{k} \varphi\right| .
$$

Adapting the proof of (2.37) but on a time space of length $|t-s|$ instead of $T$, we similarly get a bound of the form $|t-s| \varepsilon C$ instead of $T \varepsilon C$. Then we get again a majoration of the form

$$
\left|\iint_{\mathbb{R}^{2}}\left(\rho_{k}(t, x, y)-\rho_{k}(s, x, y)\right) \varphi(x, y) d x d y\right| \leq \tilde{C}_{\varphi}|t-s|,
$$


and we have again $\rho_{l} \rightarrow \rho$ in $C\left([0, T], L_{w *}^{\infty}\left(\mathbb{R}^{2}\right)\right)$ and the rest of the proof is quite similar.

The first consequence of this result is that we will obtain solutions for any block initial data (not explicite in every cases here contrary to the onedimensional case). Then by approximation of any initial data by initial blocks and the stability result, we will get existence of solutions for any initial data.

\section{Existence result}

Prior to get the existence result, let's start by discussing the constraint relation (1.6) which leads to the difficulty of defining the product $\rho \pi$ with $\pi$ a measure and $\rho$ not necessarily continuous. Indeed in the stability result, we just have a weak formulation (3.71). We expose how it is possible to define this term in a special class of solutions. To do this, we adapt the analysis done in [2]. Then, we will prove the existence result for any initial data in a weak sense and then for functions with enough regularity, we prove that we get the product $\rho \pi=\pi$ in a more classical sense.

\section{Definition of $\rho \pi$ for $\pi$ in the class $\mathcal{M L}$}

We say that $\pi$ is in $\mathcal{M L}$ if $\pi$ lies in $\mathcal{M}_{\text {loc }}\left(\left[0, \infty\left[\times \mathbb{R}^{2}\right)\right.\right.$ and if there exists $C$ such that

$$
\left|\int_{[0, \infty[} \int_{\mathbb{R}} \int_{\mathbb{R}} \phi(t, x, y) \pi(t, x, y)\right| \leq C\|\phi\|_{L_{t}^{\infty}(] 0, \infty\left[, L_{x y}^{1}\left(\mathbb{R}^{2}\right)\right)}, \quad \forall \phi \in C_{c}\left(\left[0, \infty\left[\times \mathbb{R}^{2}\right) .\right.\right.
$$

If $\pi$ is in $\mathcal{M L}$, then thanks to the density of $C_{c}\left(\left[0, \infty\left[\times \mathbb{R}^{2}\right)\right.\right.$ in $C_{c}\left(\left[0, \infty\left[, L^{1}\left(\mathbb{R}^{2}\right)\right)\right.\right.$ for $\|\cdot\|_{L_{t}^{\infty}(] 0, \infty\left[, L_{x y}^{1}\left(\mathbb{R}^{2}\right)\right)}$, we can define $<\pi, \phi>$ for $\phi \in C_{c}\left(\left[0, \infty\left[, L^{1}\left(\mathbb{R}^{2}\right)\right)\right.\right.$ by classical arguments. We obtain besides that

$$
|<\pi, \phi>| \leq C\|\phi\|_{L_{t}^{\infty}(] 0, \infty\left[, L_{x y}^{1}\left(\mathbb{R}^{2}\right)\right)}, \quad \forall \phi \in C_{c}\left(\left[0, \infty\left[, L^{1}\left(\mathbb{R}^{2}\right)\right) .\right.\right.
$$

Definition 4.1 Let $\rho \in C_{t}\left(\left[0, \infty\left[, L_{l o c}^{1}\left(\mathbb{R}^{2}\right)\right)\right.\right.$ and $\pi \in \mathcal{M L}$. Then the product $\rho \pi$ is defined as a measure by $<\rho \pi, \phi>=<\pi, \rho \phi>$ for $\phi \in C_{c}\left(\left[0, \infty\left[\times \mathbb{R}^{2}\right)\right.\right.$.

We notice that if $\pi \in \mathcal{M}_{l o c}\left(\left[0, \infty\left[\times \mathbb{R}^{2}\right)\right.\right.$ satisfies

$\int_{[0, \infty[} \int_{\mathbb{R}} \int_{\mathbb{R}}|\phi(t, x, y) \pi(t, x, y)| \leq C\|\phi\|_{L_{t}^{\infty}(] 0, \infty\left[, L_{x y}^{1}\left(\mathbb{R}^{2}\right)\right)}, \forall \phi \in C_{c}\left(\left[0, \infty\left[, L^{1}\left(\mathbb{R}^{2}\right)\right)\right.\right.$,

then $\pi \in \mathcal{M L}$ and

$$
<\pi, \phi>=\int_{[0, \infty[} \int_{\mathbb{R}} \int_{\mathbb{R}} \phi(t, x, y) \pi(t, x, y), \quad \forall \phi \in C_{c}\left(\left[0, \infty\left[, L^{1}\left(\mathbb{R}^{2}\right)\right) .\right.\right.
$$

As in [2], we have easily the following proposition which proves that the blocks of Section 2 have this regularity and then satisfy the constraint in a classical sense.

Proposition 4.2 For the sticky blocks of Section 2, the pressures $\pi_{1}$ and $\pi_{2}$ satisfy (4.92). For these blocks, we also have $\rho \in C\left(\left[0, \infty\left[, L^{1}\left(\mathbb{R}^{2}\right)\right)\right.\right.$ and then $\rho \pi=\pi$ in $\mathcal{M}_{\text {loc }}\left(\left[0, \infty\left[\times \mathbb{R}^{2}\right)\right.\right.$. 


\section{The exclusion relation $\rho \pi=\pi$ for solutions with $\pi$ in the class $\mathcal{M L}$}

Proposition 4.3 If we assume that the limit $\left(\rho, u, v, \pi_{1}, \pi_{2}\right)$ of Theorem 3.4 satisfies furthermore that $\rho_{k} \rightarrow \rho \in C_{t}\left(\left[0, \infty\left[, L_{x y}^{1}\left(\mathbb{R}^{2}\right)\right)\right.\right.$ and if we assume that $\pi_{1}, \pi_{2} \in \mathcal{M L}$, then the exclusion relations $\rho \pi_{1}=\pi_{1}$ and $\rho \pi_{2}=\pi_{2}$ hold in the sense of Definition 4.1.

Proof. We prove it for $\pi_{1}$, the proof being similar for $\pi_{2}$. We consider a sequence $\left(\rho_{k}, u_{k}, v_{k},\left(\pi_{1}\right)_{k},\left(\pi_{2}\right)_{k}\right)_{k \geq 1}$ of blocks which approximate $\left(\rho, u, v, \pi_{1}, \pi_{2}\right)$ in weak sense as in (3.72)-(3.73). Let $\varphi \in C_{c}^{\infty}\left(\left[0,+\infty\left[\times \mathbb{R}^{2}\right)\right.\right.$. We can write, since $\pi_{1} \in \mathcal{M L}$ and $\rho \in C\left(\left[0, \infty\left[, L_{l o c}^{1}\left(\mathbb{R}^{2}\right)\right)\right.\right.$,

$$
<\left(\pi_{1}\right)_{k}, \rho_{k} \varphi>-<\pi_{1}, \rho \varphi>=<\left(\pi_{1}\right)_{k},\left(\rho_{k}-\rho\right) \varphi>+<\left(\pi_{1}\right)_{k}-\pi, \rho \varphi>.
$$

On one hand, $\rho \varphi \in C_{c}\left(\left[0, \infty\left[, L^{1}\left(\mathbb{R}^{2}\right)\right)\right.\right.$ hence $<\left(\pi_{1}\right)_{k}-\pi_{1}, \rho \varphi>\underset{k \rightarrow+\infty}{\rightarrow} 0$. On the other hand, since $\left(\pi_{1}\right)_{k} \in \mathcal{M L}$,

$$
\begin{aligned}
\left|<\left(\pi_{1}\right)_{k},\left(\rho_{k}-\rho\right) \varphi>\right| & \leq C_{k} \sup _{t} \int_{\mathbb{R}}\left|\left(\rho_{k}-\rho\right) \varphi\right| d x \\
& \leq C_{k}\|\varphi\|_{L_{t, x, y}^{\infty}}^{\infty}\left\|\rho_{k}-\rho\right\|_{L_{t}^{\infty}\left(L_{l o c, x, y}^{1}\right)} .
\end{aligned}
$$

We can take for the constant $C_{k}$ the smallest one, that is to say

$$
C_{k}=\sup _{\varphi \in L_{t}^{\infty}\left(L_{x y}^{1}\right), \varphi \neq 0} \frac{\left|\int_{0}^{T} \int_{\mathbb{R}^{2}} \varphi\left(\pi_{1}\right)_{k}\right|}{\|\varphi\|_{L_{t}^{\infty}\left(L_{x y}^{1}\right)}} .
$$

We consider the linear continuous applications $f_{k}$ defined, for any $\varphi \in L_{t}^{\infty}\left(L_{x y}^{1}\right)$, by $f_{k}(\varphi)=\int_{0}^{T} \iint_{\mathbb{R}^{2}} \varphi\left(\pi_{1}\right)_{k}$. For any $\varphi \in L_{t}^{\infty}\left(L_{x y}^{1}\right)$, we have $f_{k}(\varphi) \rightarrow \int_{0}^{T} \iint_{\mathbb{R}^{2}} \varphi \pi$ and then $\left(f_{k}(\varphi)\right)_{k}$ is bounded. We apply the Banach-Steinhaus Theorem to this family of applications and get that $\sup C_{k}<+\infty$.

Therefore we get $\lim _{k \rightarrow+\infty}<\left(\pi_{1}\right)_{k}, \rho_{k} \varphi>^{k}=<\rho, \pi_{1} \varphi>=<\rho \pi_{1}, \varphi>$. Now $\left(\pi_{1}\right)_{k}=\rho_{k}\left(\pi_{1}\right)_{k} \rightarrow \tilde{\pi}_{1}$ and then $\tilde{\pi}_{1}=\rho \pi_{1}$ and the constraint $\tilde{\pi}_{1}=\pi_{1}$ becomes $\rho \pi_{1}=\pi_{1}$.

\section{Existence of solutions}

We are now able to prove the following existence theorem.

Theorem 4.4 (Existence of solutions) Let us consider initial data $\left(\rho^{0}, u^{0}, v^{0}\right)$ with regularities $\rho^{0} \in L^{\infty}\left(\mathbb{R}^{2}\right) \cap L^{1}\left(\mathbb{R}^{2}\right)$, $u^{0}, v^{0} \in L^{\infty}\left(\mathbb{R}^{2}\right) \cap B V\left(\mathbb{R}^{2}\right)$. Then there exists $\left(\rho, u, v, \pi_{1}, \pi_{2}\right)$, with regularities (1.8)-(1.10), which are solution of (1.4) with the constraints (1.5) and satisfy the bounds

$$
0 \leq \rho \leq 1, \quad \iint_{\mathbb{R}^{2}} \rho(t, x, y) d x d y \leq \iint_{\mathbb{R}^{2}} \rho^{0}(x, y) d x d y
$$




$$
\begin{gathered}
\text { essinf } u^{0} \leq u \leq \text { esssup } u^{0}, \quad \text { essinf } v^{0} \leq v \leq \text { esssup } v^{0} \\
\iint_{\mathbb{R}^{2}}\left|\partial_{x} u(t, x, y)\right| \leq \iint_{\mathbb{R}^{2}}\left|\partial_{x} u^{0}(x, y)\right|, \quad \iint_{\mathbb{R}^{2}}\left|\partial_{y} u(t, x, y)\right| \leq \iint_{\mathbb{R}^{2}}\left|\partial_{y} u^{0}(x, y)\right|, \\
\iint_{\mathbb{R}^{2}}\left|\partial_{x} v(t, x, y)\right| \leq \iint_{\mathbb{R}^{2}}\left|\partial_{x} v^{0}(x, y)\right|, \quad \iint_{\mathbb{R}^{2}}\left|\partial_{y} v(t, x, y)\right| \leq \iint_{\mathbb{R}^{2}}\left|\partial_{y} v^{0}(x, y)\right| .
\end{gathered}
$$

Furthermore, there exists $\tilde{\pi}_{1}, \tilde{\pi}_{2} \in \mathcal{M}_{\text {loc }}\left(\left[0, \infty\left[\times \mathbb{R}^{2}\right)\right.\right.$ such that the constraint (1.6) is satisfied in the weak sense (3.71).

Proof. Let $\rho_{k}^{0}, u_{k}^{0}, v_{k}^{0}\left(k \in \mathbb{N}^{*}\right)$ be the block initial data associated respectively to $\rho^{0}, u^{0}, v^{0}$ provided by Lemma 3.1. Proposition 2.12 gives $\left(\rho_{k l}, u_{k l}, v_{k l},\left(\pi_{1}\right)_{k l},\left(\pi_{2}\right)_{k l}\right)$ such that

$$
\left\{\begin{array}{l}
\partial_{t} \rho_{k l}+\partial_{x}\left(\rho_{k l} u_{k l}\right)+\partial_{y}\left(\rho_{k l} v_{k l}\right)=R_{k l} \underset{l \rightarrow+\infty}{\rightarrow} 0, \\
\partial_{t}\left(\rho_{k l} u_{k l}\right)+\partial_{x}\left(\rho u_{k l}^{2}+\left(\pi_{1}\right)_{k l}\right)+\partial_{y}\left(\rho_{k l} u_{k l} v_{k l}\right)=S_{k l} \underset{l \rightarrow+\infty}{\rightarrow} 0 \\
\partial_{t}\left(\rho_{k l} v_{k l}\right)+\partial_{x}\left(\rho u_{k l} v_{k l}\right)+\partial_{y}\left(\rho_{k l} v_{k l}^{2}+\left(\pi_{2}\right)_{k l}\right)=T_{k l} \underset{l \rightarrow+\infty}{\rightarrow \rightarrow \infty} 0
\end{array}\right.
$$

in the distributional sense. At $k$ fixed, these functions satisfy the bounds of Theorem 3.5 and we can apply it to get that, up to subsequence, and making a diagonal Cantor process, the convergence in the distributional sense $\left(\rho_{k l}, u_{k l}, v_{k l},\left(\pi_{1}\right)_{k l},\left(\pi_{2}\right)_{k l}\right) \underset{l \rightarrow+\infty}{\rightarrow}\left(\rho_{k}, u_{k}, v_{k},\left(\pi_{1}\right)_{k},\left(\pi_{2}\right)_{k}\right)$, where the obtained limit $\left(\rho_{k}, u_{k}, v_{k},\left(\pi_{1}\right)_{k},\left(\pi_{2}\right)_{k}\right)$, with regularities (1.8)-(1.10), is solution of (1.4) with the constraints $(1.5),(3.71)$, with $\left(\tilde{\pi}_{1}\right)_{k},\left(\tilde{\pi}_{2}\right)_{k} \in \mathcal{M}_{l o c}\left(\left[0, \infty\left[\times \mathbb{R}^{2}\right)\right.\right.$, and satisfies the bounds (3.67)-(3.70). Furthermore $0 \leq\left(\tilde{\pi}_{1}\right)_{k} \leq\left(\pi_{1}\right)_{k}$ and $0 \leq\left(\tilde{\pi}_{2}\right)_{k} \leq$ $\left(\pi_{2}\right)_{k}$ and these measures are bounded in $\mathcal{M}_{\text {loc }}\left(\left[0, \infty\left[\times \mathbb{R}^{2}\right)\right.\right.$. We can now apply the Theorem 3.4 to these sequences, and get, up to a subsequence when $k \rightarrow \infty,\left(\rho_{k}, u_{k}, v_{k},\left(\pi_{1}\right)_{k},\left(\pi_{2}\right)_{k}\right) \rightarrow\left(\rho, u, v, \pi_{1}, \pi_{2}\right)$, where $\left(\rho, u, v, \pi_{1}, \pi_{2}\right)$, with regularities (1.8)-(1.10), are solution of (1.4) with the constraints (1.5) and satisfy the bounds (4.93)-(4.96). Up to a subsequence, we extract $\left(\tilde{\pi}_{1}\right)_{k} \rightarrow \tilde{\pi}_{1}$ and $\left(\tilde{\pi}_{2}\right)_{k} \rightarrow \tilde{\pi}_{2}$. Since $\left(\tilde{\pi}_{1}\right)_{k}=\left(\pi_{1}\right)_{k}$, we get $\tilde{\pi}_{1}=\pi_{1}$. We operate similarly for $\tilde{\pi}_{2}$ and get (3.71).

By Proposition 4.3, we finally have the additionnal property.

Remark 4.1 Furthermore, if $\rho_{k} \rightarrow \rho \in C_{t}\left(\left[0, \infty\left[, L_{x y}^{1}\left(\mathbb{R}^{2}\right)\right)\right.\right.$ and $\pi_{1}, \pi_{2} \in \mathcal{M L}$, then the exclusion relations $\rho \pi_{1}=\pi_{1}$ and $\rho \pi_{2}=\pi_{2}$ hold in the sense of Definition 4.1. Then, we get the strong constraint in the case of blocks and for the limit of this approximation when the limit is in $C_{t}\left(\left[0, \infty\left[, L_{x y}^{1}\left(\mathbb{R}^{2}\right)\right)\right.\right.$. In the most general case, we only have a convergence in $C_{t}\left(\left[0, \infty\left[, L_{w *}^{\infty}\left(\mathbb{R}^{2}\right)\right)\right.\right.$.

\section{References}

[1] L. Barthélemy, Problème d'obstacle pour une équation quasi-linéaire du premier ordre, Ann. Fac. Sci. Toulouse Math. 9 (1988) 137-159. 
[2] F. Berthelin, Existence and weak stability for a two-phase model with unilateral constraint, Math. Models \& Methods in the Applied Sciences, 12 (2002), 249-272.

[3] F. Berthelin, Numerical flux-splitting for a class of hyperbolic systems with unilateral constraint, Mathematical Modelling and Numerical Analysis 37 (2003), no. 3, 479-494.

[4] F. Berthelin, F. Bouchut, Weak solutions for a hyperbolic system with unilateral constraint and mass loss, Annales de l'Institut Henry Poincaré, Analyse non linéaire 20 (2003), 975-997.

[5] F. Berthelin, D. Broizat, A model for the evolution of traffic jams in multilane, Kinetic and Related Models, Vol. 5, no. 4, (2012), 697-728.

[6] F. Berthelin, P. Degond, M. Delitala, M. Rascle, Model for the formation and evolution of traffic jams, ARMA, Archives for Rational Mechanics Analysis, 187 (2008), no. 2, 185-220.

[7] F. Berthelin, P. Degond, V. LeBlanc, S. Moutari, M. Rascle, J. Royer, A traffic-flow model with constraint for the modeling of traffic jams, M3AS, Mathematical Models \& Methods in Applied Science, Vol. 18, No 1 (2008) 1269-1298.

[8] F. Bouchut, Y. Brenier, J. Cortes, J.-F. Ripoll, A hierarchy of models for two-phase flows, J. of Nonlinear Science 10 (2000), 639-660.

[9] F. Bouchut, F. James, Duality solutions for pressureless gases, monotone scalar conservation laws, and uniqueness, Comm. Partial Differential Equation 24 (1999) 2173-2189.

[10] Y. Brenier, E. Grenier, Sticky particles and scalar conservation laws, SIAM J. Numer. Anal. 35 (1998) 2317-2328.

[11] D. Bresch, C. Perrin, E. Zatorska, Singular limit of a NavierStokes system leading to a free/congested zones two-phase model, Comp. Rendus Math. 352 (2014), 685-690.

[12] W. E., Y.G. Rykov, Y.G. Sinai, Generalized variational principles, global weak solutions and behavior with random initial data for systems of conservation laws arising in adhesion particle dynamics, Comm. Math. Phys. 177 (1996) 349-380.

[13] G. Gagneux, A.-M. Lefevere, M. Madaune-Tort, Une approche analytique d'un modèle black oil des écoulements triphasiques compressibles en ingénierie pétrolière, J. Méc. Théor. Appl. 6 (1987), no. 4, 547-569.

[14] E. Grenier, Existence globale pour le système des gaz sans pression, C. R. Acad. Sci. Paris Sér. I Math. 321 (1995) 171-174.

[15] L. Lévi, Problèmes unilatéraux pour des équations non linéaires de convection-réaction, Ann. Fac. Sci. Toulouse Math. 4 (1995) 593-631.

[16] L. Lévi, Obstacle problems for scalar conservation laws, ESAIM: M2AN 35 (2001) 575-593. 
[17] N. Masmoudi, P.-L. Lions, On a free boundary barotropic model, Ann. Inst. H. Poincaré Anal. Nonlinéaire 16 (1999) 373-410.

[18] C. Perrin, E. Zatorska, Free/congested two-phase model from weak solutions to multi-dimensional compressible Navier-Stokes equations, Comm. Partial Differential Equations 40 (2015), no. 8, 1558-1589. 